\title{
miR-302 regulates pluripotency, teratoma formation and differentiation in stem cells via an AKT1/OCT4- dependent manner
}

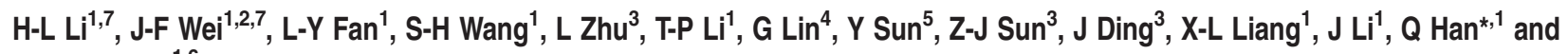 \\ R-C-H Zhao, ${ }^{* 1,6}$
}

Pluripotency makes human pluripotent stem cells (hPSCs) promising for regenerative medicine, but the teratoma formation has been considered to be a major obstacle for their clinical applications. Here, we determined that the downregulation of miR-302 suppresses the teratoma formation, hampers the self-renewal and pluripotency, and promotes hPSC differentiation. The underlying mechanism is that the high endogenous expression of miR-302 suppresses the AKT1 expression by directly targeting its $3^{\prime}$ UTR and subsequently maintains the pluripotent factor OCT4 at high level. Our findings reveal that miR-302 regulates OCT4 by suppressing AKT1, which provides hPSCs two characteristics related to their potential for clinical applications: the benefit of pluripotency and the hindrance of teratoma formation. More importantly, we demonstrate that miR-302 upregulation cannot lead OCT4 negative human adult mesenchymal stem cells (hMSCs) to acquire the teratoma formation in vivo. Whether miR-302 upregulation can drive hMSCs to acquire a higher differentiation potential is worthy of deep investigation.

Cell Death and Disease (2016) 7, e2078; doi:10.1038/cddis.2015.383; published online 28 January 2016

Human pluripotent stem cells (hPSCs), including human embryonic stem cells (hESCs) and human-induced pluripotent stem cells, have the ability to self-renew and differentiate into cells of the three germ layers, ${ }^{1,2}$ which makes such cells promising for regenerative medicine. However, upon transplantation in vivo, undifferentiated hESCs rapidly form stereotypical tumors called teratoma. ${ }^{3}$ The teratoma formation of hPSCs is a critical issue at present, ${ }^{4-6}$ and its cellular and molecular mechanisms remain largely unknown.

The remarkable self-renewal capacity of hESCs has been thought to be the parallel certain hallmarks of tumorigenesis, ${ }^{5,7}$ which requires a unique cell cycle structure that is characterized by a shortened $\mathrm{G} 1$ phase and the absence of $\mathrm{G} 1 / \mathrm{S}$ checkpoint. ${ }^{8,9}$ The absence of cell cycle inhibitors ${ }^{10,11}$ allows hESCs to proliferate rapidly and selfrenew constantly. ${ }^{8,9}$ ESCs share some cellular and molecular phenotypes with cancer cells. ${ }^{6,7,12}$ OCT4 is a core transcriptional factor for self-renewal and pluripotency in PSCs. OCT4 also express in some tumors, ${ }^{13-16}$ and possess oncogenic potential ${ }^{4,17}$ as a cell cycle promoter. ${ }^{18}$ These findings suggest that OCT4 may have important roles in the regulation of the cell cycle and tumorigenesis.
Today, it is widely recognized that in addition to proteincoding genes, microRNAs (miRNAs) are connected to the transcriptional regulatory circuitry of ESCs. ${ }^{19,20}$ miR-302 can target both positive and negative cell cycle-related genes, increase the number of cells in $S$ phase and shape an ESC-like cell cycle. ${ }^{21}$ ESCs cell-specific miRNAs including miR-302 can promote the $\mathrm{G} 1$ to $\mathrm{S}$ transition and rescue the proliferation defect in the Dgcr8 knockout ESCs. ${ }^{22}$ However, upregulation of miR-302 also suppressed CDK2 and CCND1, which simultaneously blocked the $\mathrm{G} 1$ to $\mathrm{S}$ transition in some tumor cells. ${ }^{23}$ OCT4 and SOX2 can bind to a conserved promoter region of miR-302 $2^{21}$ and affect its expression in hESCs, ${ }^{19,21}$ whereas miR-302 can induce and enhance somatic cell reprogramming to a pluripotent state..$^{24-26}$ However, the role miR-302 has in the regulation of cell cycle, pluripotency and tumorigenicity in hPSCs and hMSCs remains unclear.

In this study, we comprehensively investigated the effects of miR-302 on the biological characteristics of hPSCs and hMSCs. We demonstrate that endogenous miR-302 in hPSCs is just a contributing factor for the pluripotency and the teratoma formation through promoting $\mathrm{G} 1$ to $\mathrm{S}$ transition and

\footnotetext{
${ }^{1}$ Department of Cell Biology, Institute of Basic Medical Sciences Chinese Academy of Medical Sciences, School of Basic Medicine Peking Union Medical College, Tissue Engineering Center of Chinese Academy of Medical Sciences, Beijing, China; ${ }^{2}$ Department of Histology and Embryology, School of Basic Medical Sciences, Xuzhou Medical University, Xuzhou, China; ${ }^{3}$ Department of Obstetrics and Gynecology, Peking Union Medical College Hospital, Beijing, China; ${ }^{4}$ Institute of Reproductive and Stem Cell Engineering, Key Laboratory of Stem Cells and Reproductive Engineering, Ministry of Health, Central South University, Changsha, China; ${ }^{5}$ Department of Nuclear Medicine, Peking Union Medical College Hospital, Beijing, China and ${ }^{6}$ Peking Union Medical College Hospital, Beijing, China

${ }^{*}$ Corresponding author: Q Han or RC Zhao, Institute of Basic Medical Sciences Chinese Academy of Medical Sciences, School of Basic Medicine Peking Union Medical College, Tissue Engineering Center of Chinese Academy of Medical Sciences, Beijing 100005, China. Tel: +86 1065123207 or +86 1065125311 ; Fax: +86 10 65125311; E-mail: hanqinhanqin@126.com or zhaochunhua@vip.163.com

${ }^{7}$ These authors contributed equally to this work.

Abbreviations: hPSCs, human pluripotent stem cells; hESCs, human embryonic stem cells; ECCs, embryonal carcinoma cells; hNT-2, human Tera-2; hMSCs, human adult mesenchymal stem cells; $3^{\prime} U T R$, the $3^{\prime}$ untranslated region; NOD/SCID, immunodeficient nonobese diabetic/severe combined immunodeficiency; EB, embryoid body; RA, retinoic acid

Received 27.8.15; revised 20.10.15; accepted 20.11.15; Edited by Y Shi
} 
maintaining OCT4 at high level by directly inhibiting AKT1. However, although the expression of AKT1 was also sharply reduced and cell proliferation was accelerated by
miR-302 upregulation in hMSCs, overexpression of miR-302 did not lead OCT4 negative hMSCs to acquire the ability of tumor formation, which suggested that the a
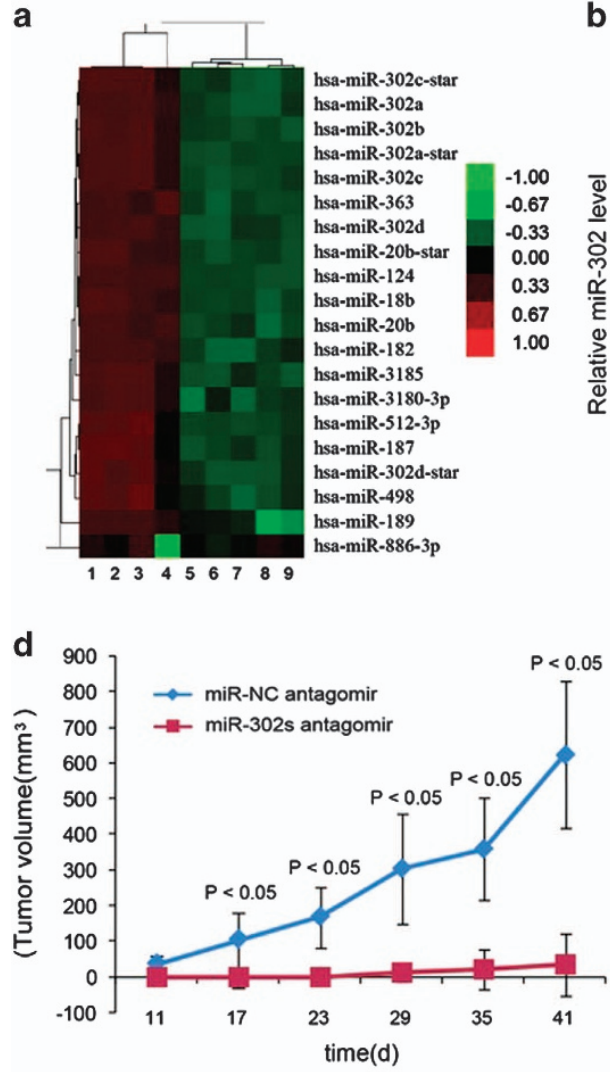

f

\begin{tabular}{cllll}
\hline Group & \multicolumn{4}{c}{ tumor weights $(g)$} \\
\hline & 1.095 & 1.282 & 1.212 & 0.884 \\
miR-NC & 0.989 & 0.865 & 0.725 & 0.808 \\
antagomir & 0.738 & 0.701 & 0.617 & 0.625 \\
& 0.532 & 0.165 & 0.127 & 0.04 \\
\hline \multirow{2}{\text{miR-302}}{} & 0 & 0 & 0 & 0 \\
antagomir & 0 & 0 & 0 & 0 \\
\hline
\end{tabular}

h

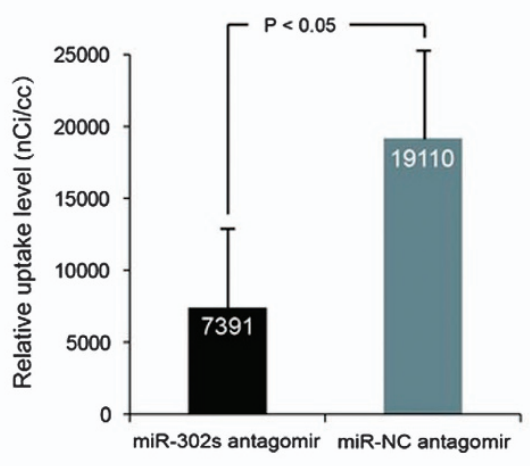

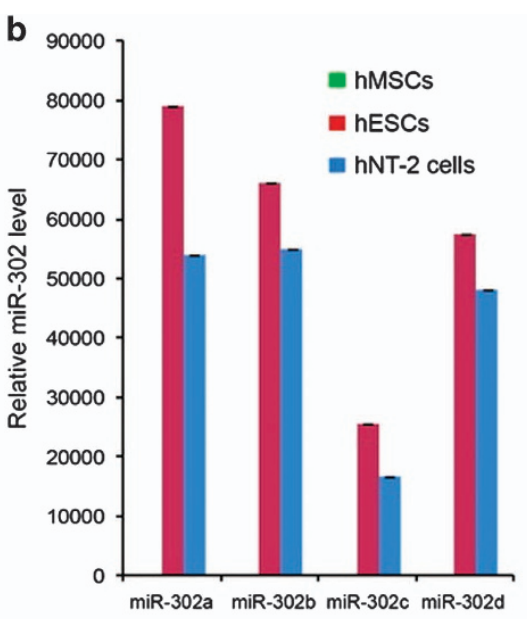

C miR-302s antagomir

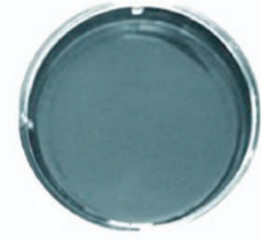

miR-NC antagomir

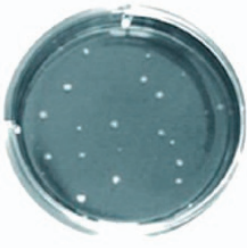

e miR-NC antagomir

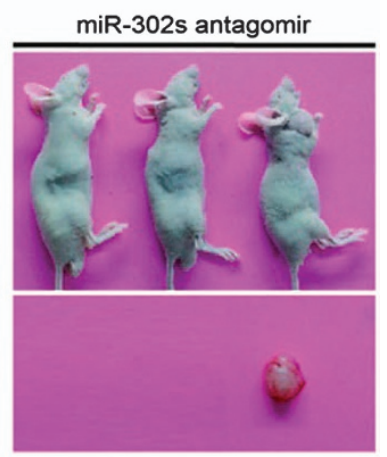

g 2, 4, 6: miR-302s antagomir; 1, 3, 5: miR-NC antagomir

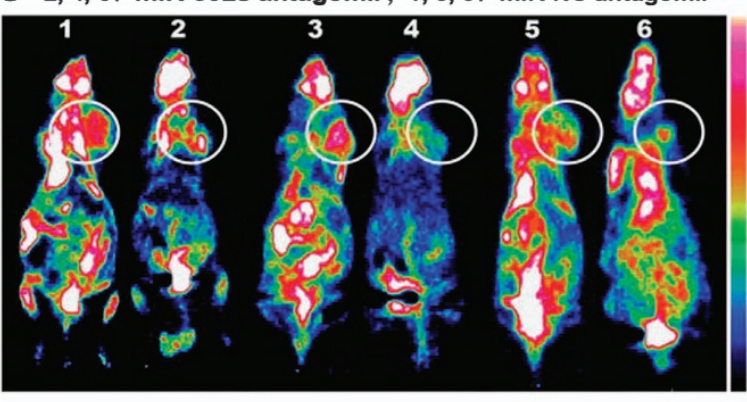

i

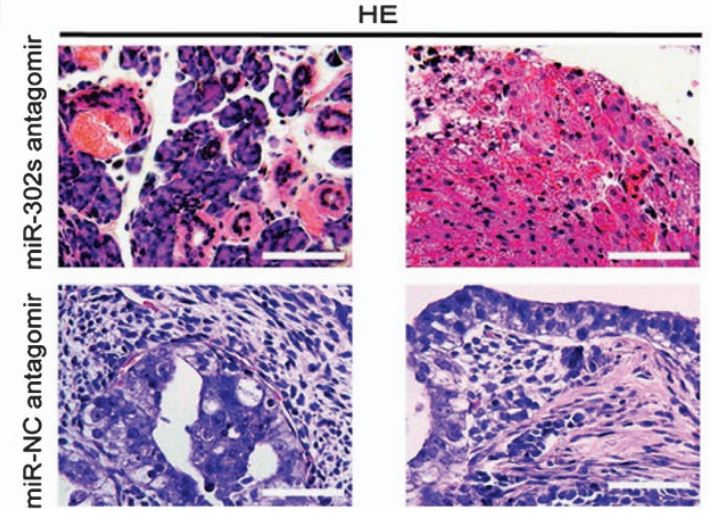


regulation of miR-302 on pluripotency and teratoma formation may be dependent on the high endogenous expression of OCT4 in cells.

\section{Results}

Downregulation of miR-302 inhibits the teratoma formation of hPSCs. ESCs and human embryonal carcinoma cells (ECCs, a malignant counterpart of hESCs) ${ }^{4}$ have overlapping metabolic signatures, ${ }^{27}$ and similar gene, protein and miRNA expression profiles, ${ }^{28,29}$ ECCs can serve as a model for studying the characteristics of ESCs. ${ }^{30}$ Human Tera-2 (hNT-2) cell is an ECC cell line that can differentiate into a variety of somatic tissues in vivo. ${ }^{31} \mathrm{We}$ and other researchers confirmed that, hESCs and hNT-2 cells have the ability to form teratoma in vivo while hMSCs do not (Supplementary Figures S1A and D). Our miRNA microarray and TaqMan qRT-PCR data revealed that the endogenous expression levels of miR-302 family were high in hESCs and hNT-2 cells but very low in hMSCs (Figures 1a and b; Supplementary Figures S1E and F and Supplementary Table S1). We presumed that high endogenous expression of miR-302 in hPSCs might be responsible for their teratoma formation. miR-302s antagomir (miR-302a, miR-302b, miR-302c and miR-302d in combination) was used to silence the endogenous miR-302s in vitro and in vivo (Supplementary Figure S2A). We found that downregulation of miR-302s dramatically abrogated the colony formation ability of hNT-2 cells in soft agar (Figure 1c). The growth of tumors in miR-302s-downregulated xenografts was gradually delayed at different time points for up to 41 days after inoculation (Figure 1d). All mice produced teratocarcinomas in the negative control group, but only $25 \%$ of mice developed teratocarcinomas from miR-302s-downregulated cells and the tumor weights were decreased by $92 \%$ at the final time point (Figures 1e and f; Supplementary Figure S2B). Small-animal PET scans showed that xenografts of miR-302s-suppressed hNT-2 cells displayed smaller volumes and lower uptake of fluorodeoxyglucose (FDG) than those of negative control-transfected cells (Figures $1 \mathrm{~g}$ and h). Differential maturation of liver and pancreatic tissue is visible in miR-302s-suppressed xenografts, which is a characteristic of well-differentiated and benign mature teratoma; while negative control cells formed mixed, poorly differentiated and malignant germ cell tumors (Figure 1i). Thus, miR-302 is able to promote the teratoma formation of hPSCs in vitro and in vivo.

Then miR-302-overexpressing lentiviral vector (lenti-miR-302s) that expresses a series of connected $\mathrm{miR}-302 \mathrm{a} / \mathrm{b} / \mathrm{c} / \mathrm{d}$ sequences was used to upregulate the expression of miR-302s in hMSCs (Supplementary Figures $\mathrm{S} 3 \mathrm{~A}$ and $\mathrm{B}$ ). In vitro anchorage-independent colony formation assay showed that no colony was formed either in miR-302soverexpressed hMSCs or negative control cells. When miR-302s-overexpressed hMSCs were delivered into 6-week-old male athymic mice (BALB/c nu/nu strain) and immunodeficient nonobese diabetic/severe combined immunodeficiency (NOD/SCID) mice, all mice did not produce teratoma (data not shown). These results suggested overexpression of the miR-302s alone is not sufficient to lead hMSCs to acquire the ability of teratoma formation.

miR-302 promotes the proliferation of pluripotent and adult stem cells. Tumor formation is closely related to cell proliferation. Thus, we next analyzed the impact of miR-302 on the proliferation of hPSCs and found that cell growth was suppressed gradually with an increase in the concentration of miR-302s antagomir (Figure 2a). Downregulation of miR-302s resulted in the growth suppression and the BrdU incorporation rate decrease in hNT-2 cells at different time points (Figures $2 b$ and d). Alkaline phosphatase (AP) staining assay showed that the inhibition of endogenous miR-302s resulted in the generation of smaller colonies from hESCs (Supplementary Figures S3C and D). In addition, upregulation of miR-302s in hMSCs accelerated cell growth and proliferation (Figures 2e and g; Supplementary Figure S3E). The expression level of proliferative marker PCNA was significantly reduced in the xenografts generated from miR-302s-suppressed hNT-2 cells (Figure $2 \mathrm{~h}$ ). These results demonstrated that miR-302 can promote cell proliferation both in pluripotent and adult stem cells.

miR-302 dominantly regulates a set of cell cycle inhibitors and promotes the $\mathbf{G 1}$ to $\mathbf{S}$ transition. Short $\mathrm{G} 1$ phase and fast $\mathrm{G} 1$ to $\mathrm{S}$ transition lead to the proliferation and teratoma formation of ESCs and iPSCs. ${ }^{5,32}$ To explore the intrinsic mechanisms underlying the regulation of teratoma formation by miR-302, we analyzed the cell cycle distribution and the expression patterns of key cell cycle regulators associated with the $\mathrm{G} 1$ to $\mathrm{S}$ transition in hESCs,

Figure 1 miR-302 downregulation inhibits the tumorigenicity of hPSCs in vitro and in vivo. (a) miRNA microarray analysis of the miRNA expression profile of $h E S C s$, hMSCs, fibroblasts and embryoid bodies. 1-2: H9, 3: chHES-22, 4-6: hMSCs, 7: BJ fibroblast, 8: D2, 9: 15-day embryoid body. Expression values are represented in shades of red and green relative to being above (red) or below (green) the median expression value across all samples (log scale 2, from -3 to +3 ). (b) miR-302a, miR-302b, miR-302c and miR-302d expression are detected by miRNA-specific qRT-PCR assays $(n=3)$. U6 was used as an internal normalization control, and data were normalized to the level of $h M S C s$. (c) Soft agar colony formation assay was performed using hNT-2 cells after transfection with miR-302s antagomir for 21 days. miR-NC antagomir-transfected hNT-2 cells was used as control. (d) miR-302s-suppressed hNT-2 cells were delivered into 6-week-old male athymic mice (BALB/c nu/nu strain) $(n=12)$. The volume of tumors was calculated and plotted and tumor growth curves were obtained. Negative control-transfected hNT-2 cells were used as control. (e) Representative image shows tumor formation when hNT-2 cells were delivered into mice (BALB/c nu/nu strain). (f) The weights of the tumors were measured at the end of time. (g) Xenografts were scanned by small-animal PET. The numbers 2, 4, 6 indicate the miR-302s-suppressed hNT-2 cells group, and 1, 3, 5 indicate the negative control-transfected hNT-2 cells group. White circles showed the location of xenografts in the mice. Fifty minutes after FDG injection, mice were anesthetized by inhalation of 2\% isoflurane in oxygen using Summit AS-1-000-7 animal isoflurane anesthesia equipment before microPET static data acquisition with continuous inhalation of isoflurane through an animal mask, and kept at $37^{\circ} \mathrm{C}$ until imaging. White and black are relative to being above (white) or below (black) the uptake median expression value across all samples. (h) Tissue uptake value was expressed as radioactive count per milliliter (nCi/cc). Interscapular BAT maximum uptake values and xenograft mean uptake values are measured from sagittal images and coronal images respectively $(n=6)$. The data are presented as the mean \pm S.D. (i) Histological analysis of xenografts was shown by hematoxylin and eosin staining. Scale bars, $100 \mu \mathrm{m}$. $P$-values were obtained using Student's $t$-test 
hNT-2 cells and hMSCs. Flow cytometry revealed that $50 \%$ of hESCs and hNT-2 cells were in S phase, while only $30 \%$ of hMSCs were in $\mathrm{S}$ phase (Supplementary Figure S4A).
Then, we suppressed the endogenous expression of miR-302s in hESCs and hNT-2 cells, and increased its expression in hMSCs. Results showed that the percentage of a

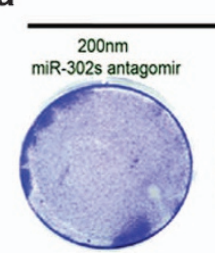

$25 \mathrm{~nm}$

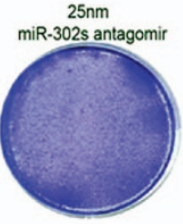

Crystal violet

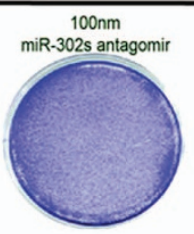

$12.5 \mathrm{~nm}$

miR-302s antagomir

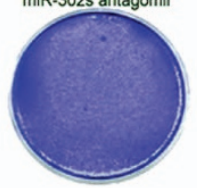

C

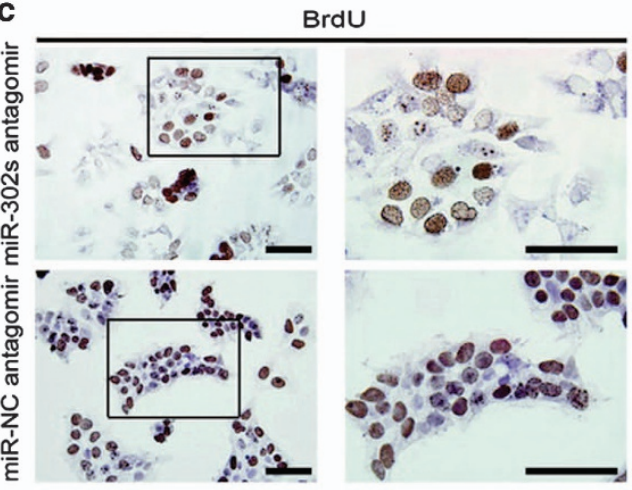

e

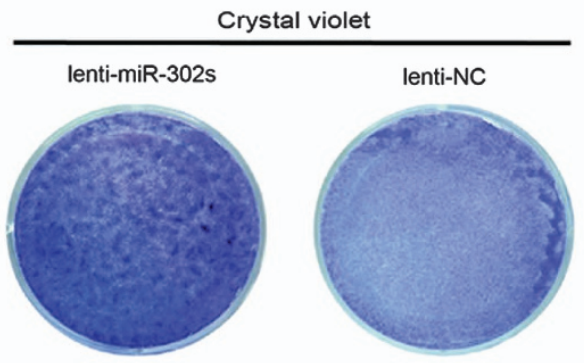

g

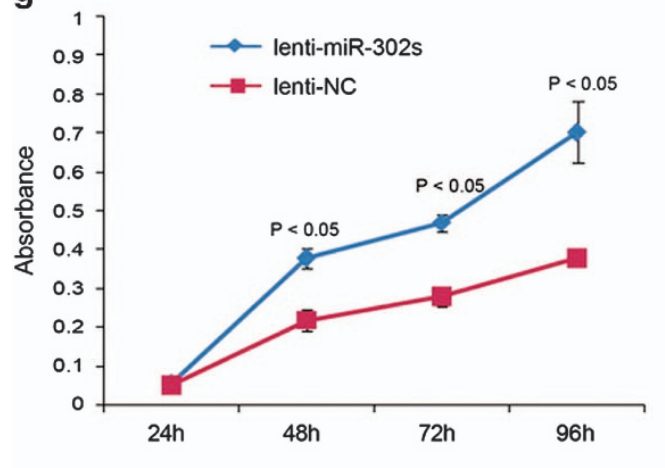

b

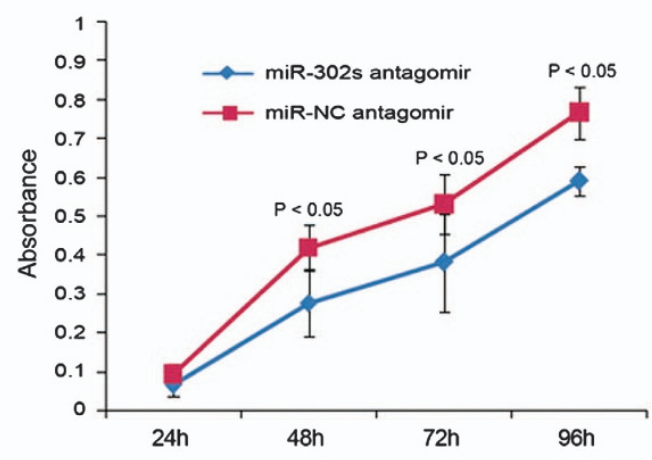

d

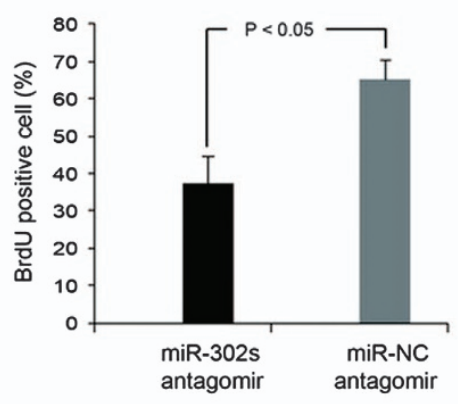

f

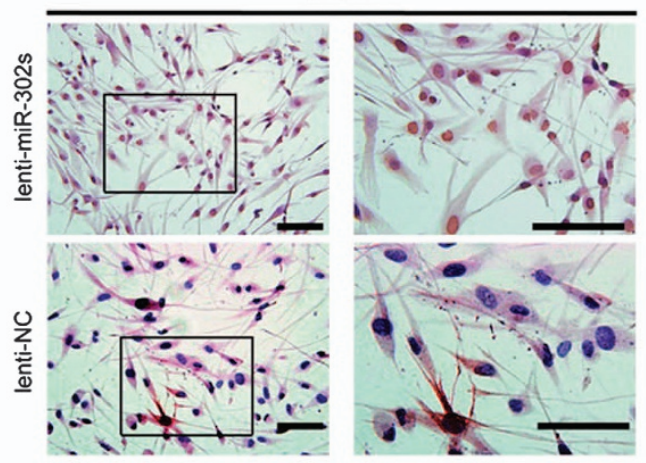

h

PCNA
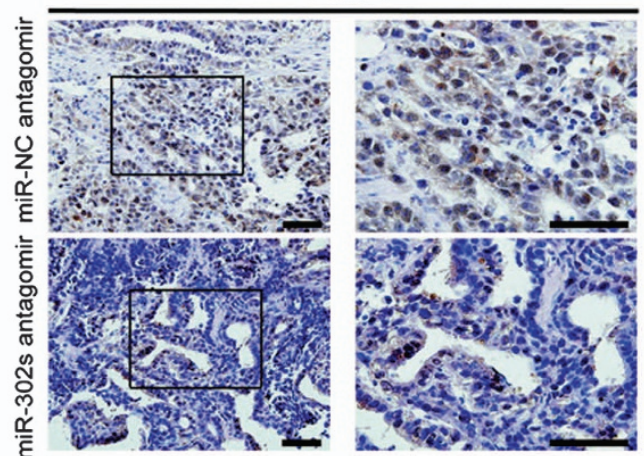
cells in S phase increased from 20.3 to $53.6 \%$ (Figure 3a) in miR-302s-overexpressed hMSCs, while it decreased from $50.4 \%$ and $51.1 \%$ to $39.6 \%$ and $41.9 \%$ in miR-302s suppressed-hESCs and -hNT-2 cells, respectively (Figure $3 b$ ). These results indicated that miR-302 promotes cell growth and proliferation partially through promoting the G1 to $S$ transition.

We then analyzed the expression levels of key regulators in the $\mathrm{G} 1$ to $\mathrm{S}$ transition and found that CDK2, CDK4, total AKT and phosphorylated AKT at S473 and P27 were at lower levels, and Cyclin D1 and P21 were almost absent in hESCs, compared with hMSCs (Figures $3 \mathrm{c}$ and e). We speculated that miR-302 might be involved in maintaining the low expression levels of these genes in hESCs. TargetScan, PicTar and Miranda suggested that many genes including both positive and negative $\mathrm{G} 1$ to $\mathrm{S}$ transition-associated regulators are candidate targets of miR-302 (Figure 3f). Previous evidence has demonstrated that the cell cycle promoters CCND1 and CDK2, and the inhibitors CDKN1A, RB1, RBL1, RBL2, LATS2 and TGFBR2, are direct targets of miR-302. ${ }^{21-23,33-35}$ Consistent with these results, we revealed that the expression levels of CCND1, CDK4, CDK6, CDKN1A and TGF $\beta$ were increased in miR-302 downregulated-hNT-2 cells and decreased in miR-302 upregulated-hMSCs (Supplementary Figures S4B and C). Our findings suggested that miR-302 can enhance proliferation through the dominant regulation of a set of cell cycle inhibitors, and result in rapid $\mathrm{G} 1$ to $\mathrm{S}$ transition.

\section{miR-302 regulates pluripotency by promoting} self-renewal and suppressing differentiation. Taking into account the positive feedback regulation involved in the $\mathrm{G} 1$ to $\mathrm{S}$ transition, self-renewal and pluripotency in $\mathrm{hPSCs}, 5,36,37$ we further assessed the role of miR-302 in self-renewal and pluripotency. According to the Protocol Exchange (Patel S, Pine S, Rameshwar P. Noble Agar Assay for Self-Renewal. Protocol Exchange 2013), single-cell clonogenic assay also indicated the self-renewal ability of stem cells. As mentioned above, our single-cell clonogenic assay revealed that downregulation of endogenous miR-302s significantly inhibited the self-renewal (Figure 1c). Immunofluorescence analysis further indicated that the expression of self-renewal marker SSEA4 was decreased in miR-302s_-downregulated hPSCs (Figure 4a).

Self-renewal involves proliferation with a concomitant suppression of differentiation. ${ }^{8}$ Therefore, to further analyze the effects of miR-302 on the modulation of differentiation, hESCs were subjected to EB formation and then placed back into ESC conditions. shRNA was used to suppress endogenous expression of miR-302s and to investigate the function of miR-302 on pluripotency and differentiation of hPSCs. Results showed that downregulation of endogenous miR-302s resulted in fewer AP-positive colonies in hESCs (Figures $4 \mathrm{~b}$ and $\mathrm{c}$ ). We next investigated whether miR-302 has a similar role in hNT-2 cells. Retinoic acid (RA) was employed to induce the differentiation. ${ }^{21}$ Results showed that most endoderm- and mesoderm-associated markers were suppressed while ectodermal and neural differentiationrelated genes were upregulated in miR-302s-downregulated hNT-2 cells (Figure 4d), which accompanied with a flattened cell morphology indicating the differentiation of cells (Figure 4e). These results showed that the inhibition of miR-302 negatively impacted self-renewal and pluripotency and promoted the differentiation of hPSCs.

miR-302 can directly target and regulate the expression of AKT1 in pluripotent and adult stem cells. AKT1 has been implicated to be an important regulator of self-renewal and pluripotency. ${ }^{38,39}$ We found that miR-302 could regulate the expression of AKT1 (Supplementary Figures S4B and C). Thus, we speculated that AKT1 might be one of the direct targets of miR-302 that controls the self-renewal and pluripotency of hPSCs. Bioinformatics analysis showed that there is a 7-bp sequence within the 3'UTR of AKT1 that is complementary to the sequence of miR-302 (Figure 5a). To further validate whether miR-302 can directly target AKT1, we designed dual luciferase reporter gene vectors containing either wild type (AKT1-WT) or mutated (AKT1-MUT) putative 3'UTR sequences for miR-302-binding (family 'seed sequence') and inserted them into the 3'UTR of the luciferase genes. Results displayed that the overexpression of miR-302 significantly suppressed $>58 \%$ of the reporter luciferase activity of the AKT1-WT reporter plasmid but not that of the AKT1-MUT reporter plasmid, suggesting a direct targeting relationship between miR-302 and the 3'UTR of AKT1 (Figure 5b). qRT-PCR and western blot analyses showed that miR-302 can regulate the expression of total AKT1 and phosphorylated AKT1 mainly at S473 locus (Figures $5 \mathrm{c}$ and d; Supplementary Figures S4B and C) both in hNT-2 and hMSCs. These results strongly confirmed that miR-302 can directly target AKT1 and suppress its expression.

miR-302 contributes to the pluripotency and teratoma formation of hPSCs by maintaining OCT4 expression via suppressing AKT1. To clarify whether miR-302 mediates pluripotency and differentiation of hPSCs through the regulation of AKT1, we first analyzed the expression of

\footnotetext{
Figure 2 miR-302 promotes the proliferation of pluripotent and adult stem cells. (a) Crystal violet staining assessed the cell growth in different miR-302s antagomir concentration-treated hNT-2 cells. miR-NC antagomir was used as negative control. (b) The growth curve at different time points was obtained by the cell counting Kit-8. Data are presented as mean \pm S.D. $(n=8)$. (c) Cell proliferation was compared by BrdU incorporation rates as revealed by immunocytochemical staining in miR-302s-downregulated hNT-2. Scale bars, $100 \mu \mathrm{m}$. (d) Quantitative analysis of immunocytochemical staining for BrdU positive cells. BrdU positive cells were counted under a high power field and normalized by the total cell number. (e) The cell growth in miR-302s-upregulated hMSCs was assessed by crystal violet staining. miR-302s: miR-302-upregulated hMSCs by a miR-302-overexpressing lentiviral vector (miR-302a, miR-302b, miR-302c and miR-302d in combination), EGFP: a scrambled sequence infected hMSCs. (f) Immunocytochemical staining was used to detect the incorporation rates of BrdU in miR-302s-overexpressed hMSCs. Scale bars, $100 \mu \mathrm{m}$. (g) The growth curve of miR-302s-overexpressed hMSCs at different time points was obtained by the cell counting Kit-8 assay. Lentivector expressing a scrambled sequence was used as a control. (h) The expression level of the proliferative index PCNA was investigated in the xenografts using an immunohistochemical staining method. Scale bars, $100 \mu \mathrm{m}$. $P$-values were obtained using Student's $t$-test
} 
miR-302 during embryoid body (EB) formation. ${ }^{20}$ We found that miR-302 was gradually downregulated during EB differentiation (Figure 6a), while at the same time, AKT1 was gradually upregulated, OCT4 and SOX2 were downregulated (Figure 6b). The positive correlation between miR-302 and OCT4, and negative correlation between miR-302 and AKT1 suggested that miR-302 may enhance the expression of pluripotency regulators
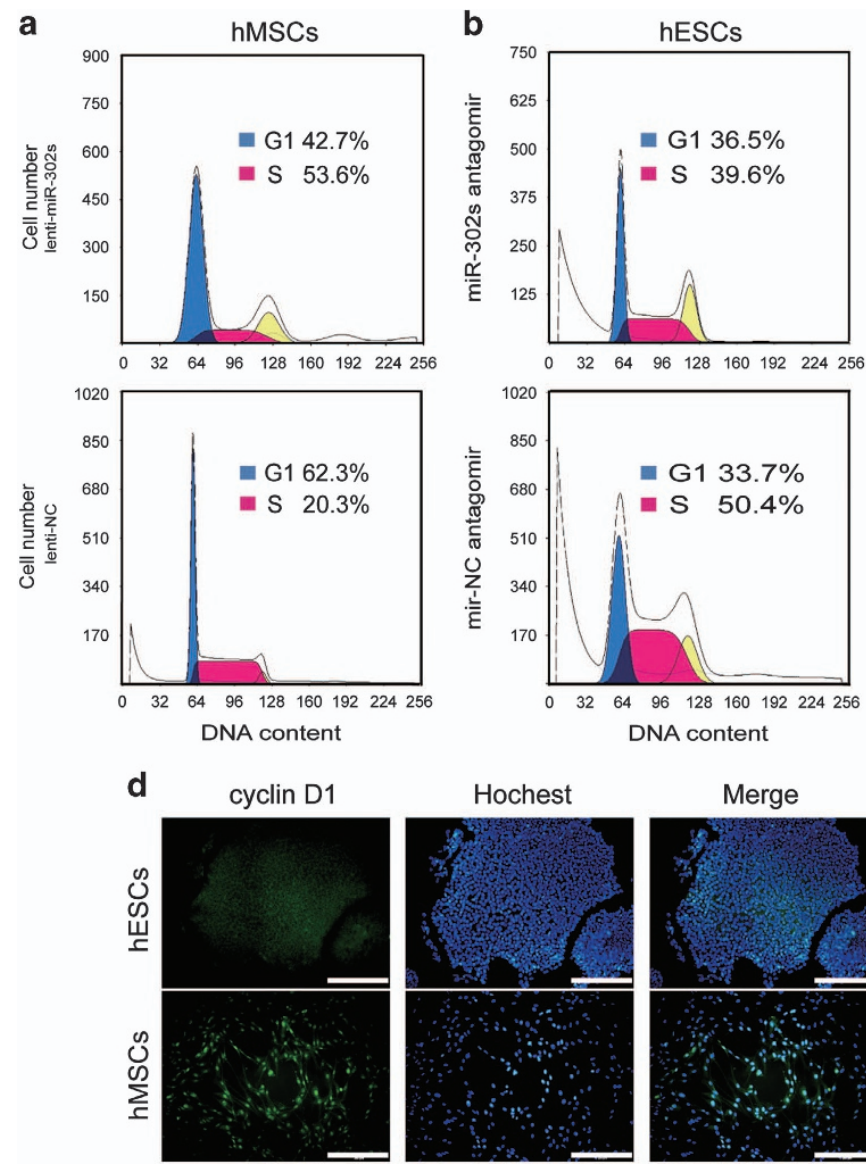

Akt1
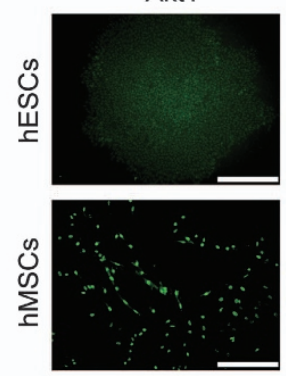

e
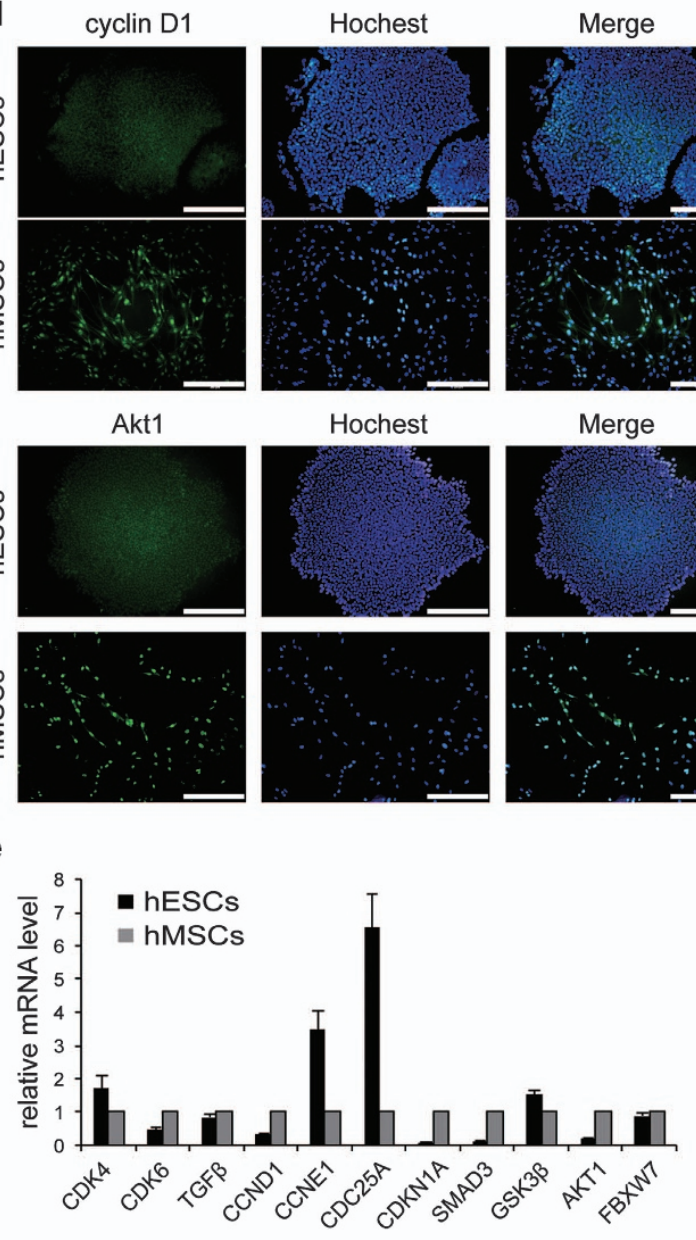

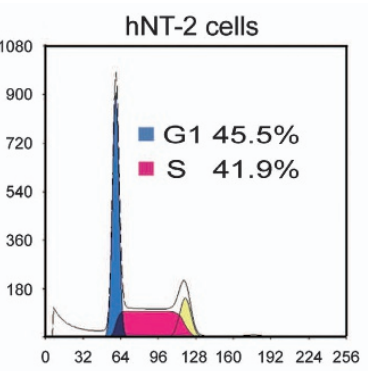

c
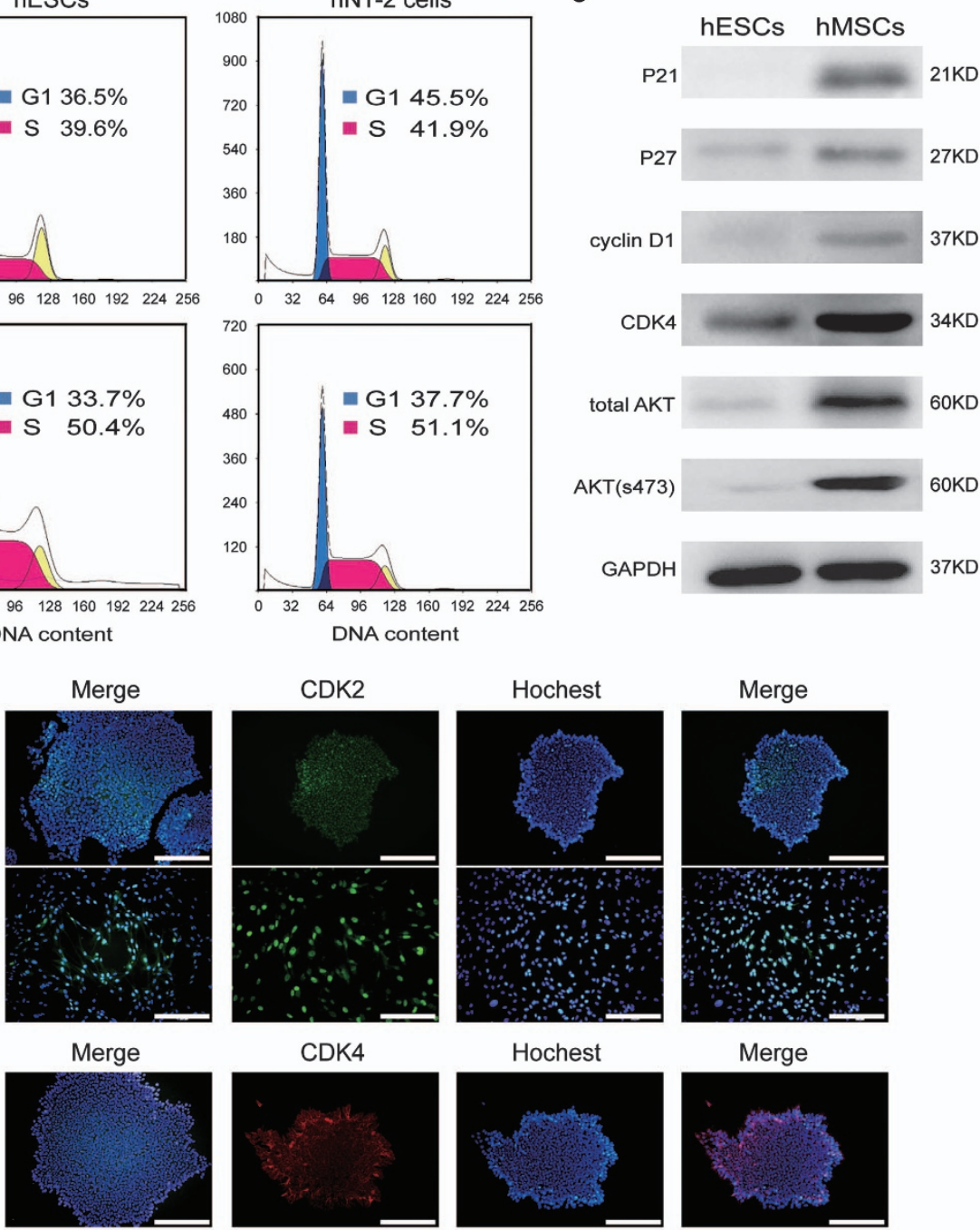

CDK4

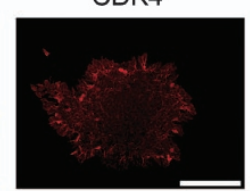

Hochest

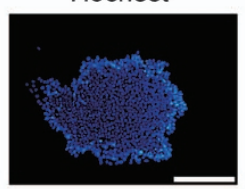

Merge
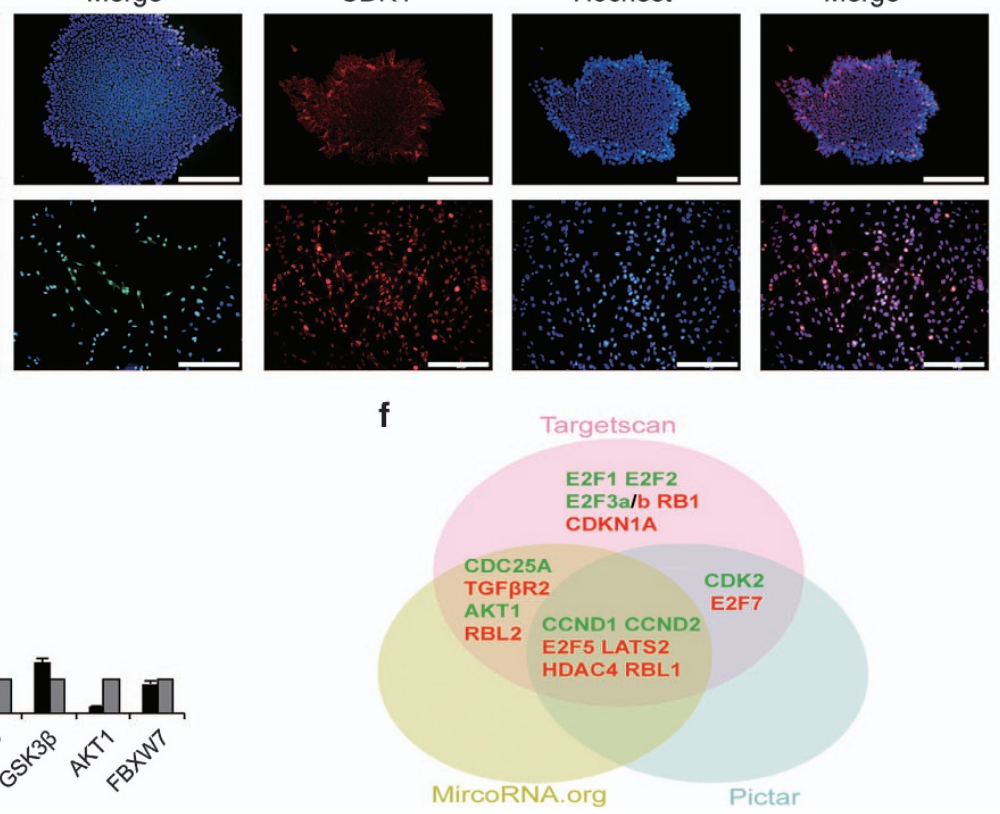
through suppressing AKT1. To clarify this hypothesis, the AKT1-overexpressing vectors were transfected into the hNT-2 cells. Results showed that OCT4 was decreased in AKT1-overexpressed hNT-2 cells, while its expression was increased in AKT1 suppressed-hNT-2 cells and was accompanied with morphological changes similar to the effect of miR-302 on the expression of AKT1 and OCT4 (Figures 6c and e). In addition, the upregulation of miR-302 in AKT1-overexpressed hNT-2 cells could inhibit AKT1 and rescue the expression of OCT4. Furthermore, miR-302 upregulation could not significantly change the expression level of OCT4 in siAKT1-transfected hNT-2 cells (Figures 6c and d), while repression of miR-302 led to the opposite effects. These results strongly demonstrated that miR-302 can maintain the expression of OCT4 at high level, at least in part by repressing AKT1. In addition, the expression of OCT4 was markedly reduced in teratoma generated from miR-302downregulated hNT-2 cells (Figure 7a). Moreover, the expression of miR-302 is very low or almost undetectable in highly differentiated patient-derived teratoma as compared with hNT-2 cells generated malignant teratoma xenografts (Figure 7b). Similarly, the expression of OCT4 was also undetectable in these 12 highly differentiated teratoma patient's specimens (Figure 7c). Therefore, our findings revealed that high expression levels of endogenous miR-302 in hPSCs are beneficial for the pluripotency and tumorigenicity of hPSCs by maintaining OCT4 at high level through suppressing AKT1 (Figure 7d). Overexpression of miR-302 also significantly decreased AKT1 expression in hMSCs (Supplementary Figure S5A), whereas OCT4 always maintained at undetectable level regardless of the expression changes of miR-302 and AKT1 in hMSCs (Supplementary Figures S6A and B).

\section{Discussion}

miRNAs are important epigenetic regulatory molecules, have been demonstrated to participate in many biological processes. ${ }^{40}$ Here, we demonstrate that miR-302 has an important role in regulating cell proliferation, self-renewal, pluripotency, differentiation and teratoma formation. miR-302 can promote the proliferation and self-renewal both in hPSCs and hMSCs through dominantly regulating a set of cell cycle inhibitors and accelerating the $\mathrm{G} 1$ to $\mathrm{S}$ transition. High expression of endogenous miR-302 in hPSCs is a contributing factor for the pluripotency and teratoma formation through maintaining OCT4 at high level by directly inhibiting AKT1. However, upregulation of miR-302 cannot lead OCT4 negative hMSCs to acquire the teratoma formation.
hPSCs have strong teratoma formation ability and high endogenous expression of miR-302. ${ }^{24,41-44}$ However, there is a big controversy about what role miR-302 has in the regulation of stemness, cell proliferation, tumorigenesis and differentiation. In some cases, miR-302s have been shown to produce iPSCs from human somatic cells and cancer cells. ${ }^{25,26,45-47}$ Alternatively, miR-302s also have been reported to inhibit the tumorigenicity of hPSCs and various cancer cells. ${ }^{23,48,49}$ We revealed that downregulation of miR-302 significantly decreased the rate of tumor formation and reduced the tumor volume in hPSCs.

Previous studies have indicated that tumorigenicity is closely related to self-renewal and cell cycle. ${ }^{5,7-9,33,35}$ To date, both positive and negative cell cycle-associated factors have been reported as direct targets of miR-302. ${ }^{21-23,33-35} \mathrm{We}$ and several reports demonstrated that miR-302s downregulated CDK2, CDK4, AKT1, P27, cyclin A, E2F-1 and cyclin $\mathrm{D} 1^{21,23,50}$ in stem cells, while others reported that the levels of CDK2, cyclin A and E2F-1 were unaffected in miR-302-367overexpressed HeLa and $\mathrm{SiHa}$ cells. ${ }^{51}$ We found a marked suppression of cell cycle progression in miR-302-suppressedhNT-2 cells, while an increase in the number of S phase cells in miR-302-overexpressed hMSCs. This result coincides with recent report that $\mathrm{miR}-302$ induces proliferation in human adipose tissue-derived MSCs. ${ }^{52}$ Thus, our data suggest that the negative cell cycle regulators are dominant targets of miR-302 in ESCs, hNT-2 cells and hMSCs.

The ectopic expression of miR-302 is able to reprogram and promote human somatic cells to ESC-like cells. ${ }^{53,54}$ Recently, the role of miR-302 in nerve development has been reported. NPCs exhibit enhanced proliferation, precocious differentiation and decreased cell death ${ }^{55}$ or survival ${ }^{56}$ in miR-302s mutant embryos. More recently, miR-302 has been reported to be capable of regulating Brg1 chromatin remodeling complex composition in hESCs, and subsequently regulating pluripotency by positively influencing mesendodermal differentiation. ${ }^{57}$ These findings suggested a complicated relationship between pluripotency and differentiation related to miR-302. We found that inhibition of miR-302 causes a significant decrease in selfrenewal ability and promotes differentiation accompanied by downregulation of OCT4, suggesting that high level of endogenous miR-302 may be responsible for the maintenance of self-renewal and pluripotency in hPSCs.

Some reports implied that abnormal activation of AKT negatively regulates HSC stemness. ${ }^{58-60}$ The functions of AKT in stem cells ${ }^{59,61,62}$ have been investigated, but its precise role in the mechanism by which AKT modulates stemness and differentiation is not yet clarified. Here, we observed that in addition to directly targeting cell cycle-associated genes, miR-302 also affects the expression

\footnotetext{
Figure 3 miR-302 regulates cell cycle progression by promoting the $\mathrm{G} 1$ to $\mathrm{S}$ transition. (a) Flow cytometry analysis of the cell cycle distribution in hMSCs when exogenous expression of miR-302 was upregulated. A lentiviral vector expressing a scrambled sequence was used as a control. (b) Flow cytometry analysis of the cell cycle distribution in hESCs and hNT-2 cells when endogenous expression of miR-302s was suppressed by miR-302s antagomir. Negative control oligonucleotide antagomir was transfected as control. (c) Western blot analysis in hESCs and hMSCs of the expression levels of key regulators involved in the G1 to S transition; GAPDH was used as a loading control. (d) Immunofluorescence detected the expression of proteins by Hoechst 33342 counterstaining. Scale bars, $200 \mu \mathrm{m}$. (e) qRT-PCR measured the mRNA expression of key regulators involved in the $\mathrm{G} 1$ to $\mathrm{S}$ transition. GAPDH was used as an internal normalization control. Data are presented as mean \pm S.D. $(n=3)$ and are representative of three independent experiments. (f) Targetscan, PicTar and Miranda predicated the cell cycle-associated candidate targets of miR-302. Green indicated positive regulators, red indicated negative regulators
} 
of total AKT1 and phospho-AKT mainly at S473 locus. We confirmed that AKT1 is a direct target of miR-302 in the regulation of pluripotency and differentiation of hPSCs.
Recently, AKT was found to phosphorylate OCT4, which facilitated OCT4's stability, nuclear localization and interaction with SOX2, subsequently promoted the transcription of OCT4 a

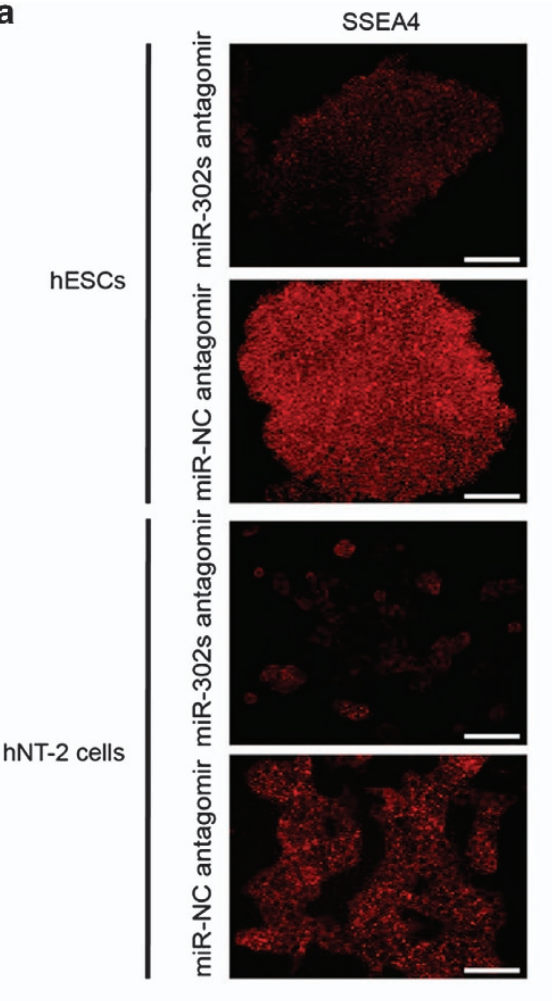

b
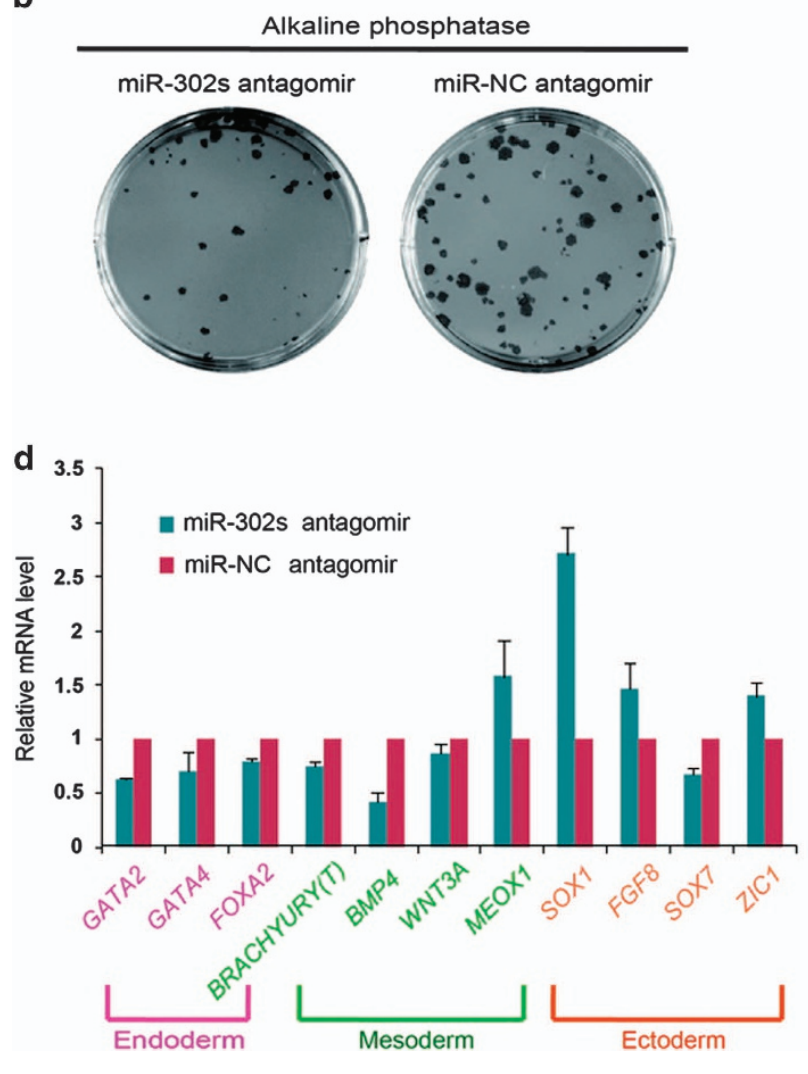

Hoechest
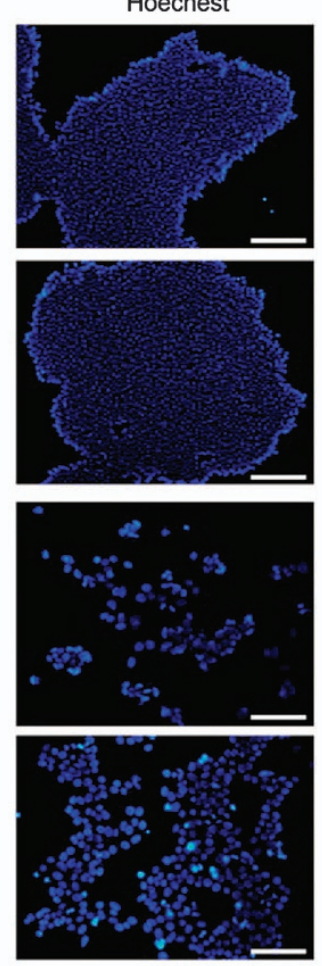

c

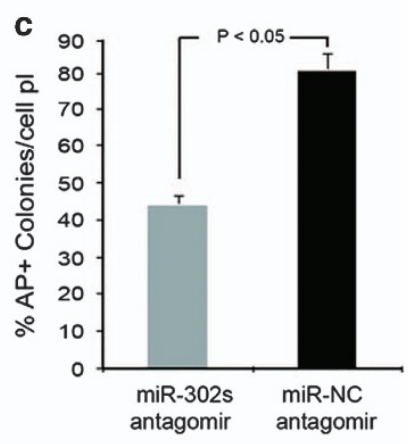

e

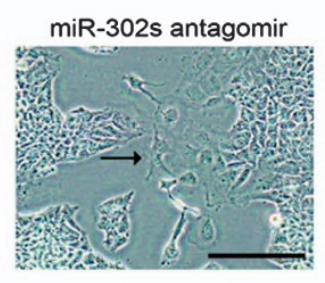

miR-NC antagomir

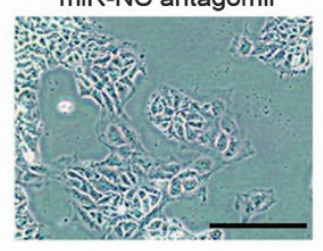


and NANOG. On the other hand, Akt phosphorylates OCT4 and KLF4, promoting their degradation via the ubiquitin-proteasome system. ${ }^{63}$ More recently, AKT1 was found to boost the function of differentiation factor SATB1 yet attenuate OCT4/KLF4 activity by phosphorylating SATB1 in ECCs. ${ }^{63}$ Consistent with this report, we found that downregulation of endogenous miR-302s or overexpression of AKT1 significantly decreased the Oct4's protein level, inhibited the self-renewal and promoted differentiation in hPSCs. Upregulation of miR-302 in AKT1-overexpressed hPSCs could inhibit AKT1 and rescue the protein level of OCT4. These results revealed that miR-302 indirectly regulates OCT4 protein level by suppressing AKT1 and subsequently avoiding OCT4's degradation.

In addition, OCT4 which is continuously required to maintain ESC pluripotency, has been identified to be expressed in some tumors $^{13,14,64,65}$ and is taking part in the regulation of the cell cycle by accelerating the G1 to $S$ transition. ${ }^{18}$ We found that OCT4 was markedly reduced in teratoma generated from miR-302-downregulated hNT-2 cells, which is in agreement with our results in vitro. Importantly, in highly differentiated teratoma of patients, which are more common clinically, the expression of miR-302 is very low or even undetectable, and the expression of OCT4 is still undetectable.

In summary, our findings first uncover that miR-302 indirectly regulates OCT4 by suppressing AKT1, which provides hPSCs two characteristics related to their potential for clinical applications: the benefit of pluripotency and the hindrance of teratoma formation. Overexpression of miR-302 did not lead OCT4 negative hMSCs to express OCT4 and acquire the teratoma formation, which suggested that the regulation of miR-302 on pluripotency and teratoma formation by direct suppression of AKT1 may be dependent on the high endogenous expression of OCT4 in cells. Whether upregulation of miR-302 can drive hMSCs to acquire a higher differentiation potential is worthy of deep investigation.

\section{Materials and Methods}

Cell lines and cell culture. hESCs cell lines H9 (Wicell, Madison, WI, USA) and chHES-22 were grown on mitomycin C (Sigma Aldrich, St. Louis, MO, USA) inactivated mouse embryonic fibroblasts (MEFs) in medium containing Dulbecco's modified Eagle's medium F-12 (DMEM; Gibco, Raritan, NJ, USA) supplemented with 20\% knockout serum replacement (Gibco), $1 \mathrm{mM}$ L-glutamine (Gibco), $0.1 \mathrm{mM}$ $\beta$-mercaptoethanol (Gibco), 1\% nonessential amino acids (Gibco) and $4 \mathrm{ng} / \mathrm{ml}$ human basic fibroblast growth factor (bFGF) (Sigma Aldrich). One to two passages before beginning our experiments, $h E S C s$ were transferred to Matrigel (BD Biosciences)-coated plates with mTeSR (Stem Cell, Vancouver, British Columbia, Canada). Differentiation through EB formation was induced by collecting $\mathrm{hESCs}$ and culturing them in suspension with hESC medium without bFGF. The hNT-2 cell line was obtained from the cell culture center of the Institute of Basic Medical Sciences of the Chinese Academy of Medical Sciences and the School of Basic Medicine of the Peking Union Medical College. hNT-2 cells were cultured according to the literature. ${ }^{23} \mathrm{Bj}$ (ATCC, Maryland Rockefeller, MD, USA, CRL-2522) and D2 fibroblasts were cultured in medium consisting of DMEM supplemented with $10 \%$ fetal bovine serum, and digested using trypsin-EDTA (Gibco). Cell cultures were maintained at $37^{\circ} \mathrm{C}$ in a humidified incubator with $5 \% \mathrm{CO}_{2}$. hNT-2 cells gave rise to embryonic bodies (EBs) in suspension culture. Differentiation by forming $\mathrm{EB}$ suspension was performed in hNT-2 cells as report. ${ }^{20}$

Isolation and expansion of human MSCs. hMSCs were isolated from adipose tissues that were obtained from patients undergoing tumescent liposuction according to procedures approved by the Ethics Committee at the Chinese Academy of Medical Sciences and Peking Union Medical College. Human specimens were digested in collagenase $P$ (Roche, Mannheim, Germany) for $30 \mathrm{~min}$ at $37^{\circ} \mathrm{C}$ and centrifuged at $300 \times \mathrm{g}$ for $5 \mathrm{~min}$. Then, the pellet was suspended in media consisting of DF12 (Gibco) supplemented with $5 \%$ MSC fetal bovine serum (Gibco). After $6 \pm 1$ days in culture at $37^{\circ} \mathrm{C}$ in a humidified incubator with $5 \% \mathrm{CO}_{2}$, adherent cells were selected for phenotypic analysis of hMSCs using flow cytometry. The expression of CD73, CD90 and CD105, together with a lack of expression of CD11b, CD14, CD34, CD45, CD19 and HLA-DR cell population was defined as mesenchymal stem cells (Supplementary Figures S6). ${ }^{66}$

Expression profiling with microarrays. Microarray experiments were conducted according to the manufacturer's instructions (www.capitalbio.com). Total RNA was prepared with TRIzol Reagent (Invitrogen, Paisley, UK). miRNA was extracted by using a mirVana miRNA Isolation Kit (Ambion, Austin, TX, USA). RNA integrity was determined by $1 \%$ formaldehyde denaturing gel electrophoresis. miRNA profiling was performed using GeneChip miRNA Array version 2.0 (Affymetrix, Santa Clara, CA, USA). Eukaryotic Hybridization Control Kit, Hybridization, Wash and Stain Kit are used to perform Hybridization. After labeling, the samples were hybridized on Affymetrix Gene-Chip miRNA arrays, washed, stained and scanned according to the manufacturer's instructions, the images obtained were then analyzed with LuxScan 3.0 software (both from CapitalBio, Beijing, China). Raw data were performed background adjustment, normalized using the rank invariant gene, and filtered for significant expression on the basis of negative control beads.

Lentiviral preparation and infection. Lentivirus production and titering were carried out according to the protocols from invitrogen (http://www.invitrogen.com. $\mathrm{cn)}$. The cells were passaged in every 2 days using $0.05 \%$ trypsin-EDTA to single cell and plated in $2 \mathrm{ml}$ aliquots per well in six-well plates at multiplicity of infection (MOI) 30. After the cells were infected with the miR-302 overexpressing GFP-labeled lentiviral vector (lenti-miR-302s), GFP-positive cells were sortinged by FACSCalibur (BD). The same GFP-labeled lentiviral vector expressing a scrambled sequence (lentiNC) with no homology to the human genome was used in parallel as a control.

RNA extraction and real-time PCR assays. Total RNA was extracted from cultured cells or freshly iced teratoma tissues with TRIzol Reagent (Invitrogen). The mRNA levels were assayed with the primer sets (see Supplementary Table S2 in the Supplementary Material) in an Applied Biosystems Fast Real-Time PCR System (Applied Biosystems, Foster, CA, USA) according to the manufacturer's instructions. miRNA was extracted with the miRVana Isolation Kit (Ambion) as described by the manufacturer. The miRNA levels were assayed by TaqMan Small RNA Assays using probe sets or SYBR green-based stem-loop technology (see Supplementary Table S3 in the Supplementary Material) designed to detect and quantify mature miRNAs (Invitrogen); the procedure was done according to the manufacturer's instructions.

\footnotetext{
Figure 4 Downregulation of miR-302 suppresses self-renewal and promotes differentiation. (a) Immunofluorescence staining of SSEA4 in hESCs and hNT-2 cells when endogenous miR-302 was inhibited by miR-302s antagomirs. The negative control oligonucleotide antagomir was transfected as a control. Scale bars, $200 \mu \mathrm{m}$. (b) miR-302sdownregulated hESCs were cultured in non-adherent conditions to form EBs. EBs were then dissociated and plated back in ESC culture conditions at day 15. ESC-like colonies were analyzed by AP staining after 6 days under EB to ESC conditions. (c) The numbers of AP-positive ESC-like colonies were calculated under EB to ESC conditions in miR-302s-downregulated hESCs. Negative control oligonucleotide antagomir was transfected as control. Data are presented as mean \pm S.D. $(n=5)$ and are representative of three independent experiments. (d) hNT-2 cells were treated with vehicle or $1 \mathrm{mM}$ retinoic acid (RA) for 4 days and the expression levels of lineage-specific markers were determined by qRT-PCR; GAPDH was used as an internal normalization control. Data are presented as mean \pm S.D. $(n=3)$ and are representative of three independent experiments. (e) Morphological changes of hNT-2 cells were investigated after treatment with vehicle or $1 \mathrm{mM}$ retinoic acid (RA) for 4 days in miR-302s antagomir-transfected hNT-2 cells and negative control cells. Scale bars, $100 \mu \mathrm{m}$. P-values were obtained using Student's $t$-test
} 

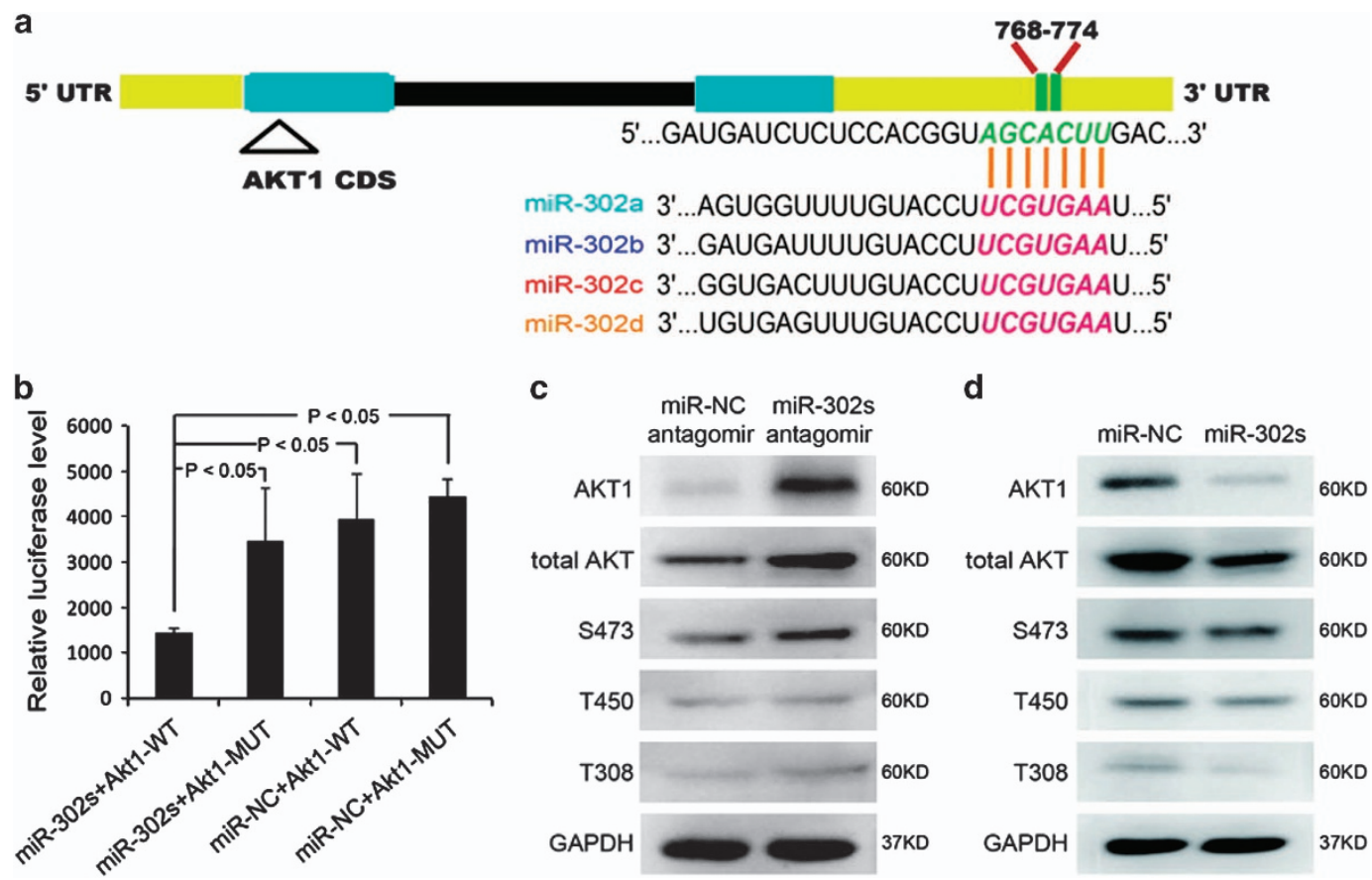

Figure 5 miR-302 represses the expression of AKT1 by targeting its $3^{\prime} U T R$. (a) Bioinformatics analysis showed that there is a 7-bp sequence in the $3^{\prime} \mathrm{UTR}$ of AKT1 that is complementary to the seed sequence of miR-302. (b) Luciferase activity was assessed in miR-302s mimic-transfected $293 \mathrm{~T}$ cells. Negative control oligonucleotide was used as control. Data are presented as mean \pm S.D. $(n=3)$ and are representative of three independent experiments. WT, wild type; MUT, mutant-type. (c, d) Western blot detected the expression levels of total AKT, AKT1 and phospho-AKT in miR-302s-antagomir-transfected hNT-2 cells and miR-302s-upregulated hMSCs. A negative control oligonucleotide was transfected as control, and GAPDH was used as a loading control

Western blot analysis. After washing twice with PBS, proteins were extracted with radioimmunoprecipitation lysis buffer with PMSF (Beyotime, Shanghai, China) and quantified by the BCA Protein Assay Kit (Beyotime). Lysates were electrophoresed on a 10\% SDS-PAGE gel and electrophoretically transferred to a polyvinylidene difluoride membrane. The membranes were blocked in Tris-buffered saline with $5 \%$ milk and $0.1 \%$ Tween. The blots were probed with CDK4, Cyclin D1 (1 : 200; all from Santa Cruz Biotechnology, Inc., Delaware, Santa Cruz, CA, USA), P21, P27, total AKT, AKT1, Phospho-AKT (S473, T450, T308), OCT4, SOX2 (1: 1000; all from Cell Signaling Technology, Danvers, MA, USA) and GAPDH (Sigma Aldrich) at $4{ }^{\circ} \mathrm{C}$ overnight and revealed with anti-mouse or anti-rabbit horseradish peroxidase-conjugated secondary antibodies (1:2000; ZSbio). Antibody-antigen complexes were detected using the chemiluminescent ECL reagent (Millipore, Billerica, MA, USA). Western blot images were acquired using an Alpha Innotech FluorChem SP Imaging System (Alpha Innotech, San Jose, CA, USA).

Dual luciferase reporter assay. An 80-100 bp synthetic fragment of gene $3^{\prime} \mathrm{UTR}$ containing the predicted seed match site or the mutant site technology (Supplementary Table S4) was inserted between the Notl and Xbal cleavage sites of the pRL-TK report vector (Promega, Southampton, UK) downstream from the renilla luciferase reporter gene. In all, $5 \times 10^{4} 293 \mathrm{~T}$ cells per well in 24-well plates were co-transfected with $1 \mu \mathrm{g} \mathrm{pRL-TK}$ vector with or without the synthetic fragment of the AKT1 $3^{\prime}$ UTR and $0.1 \mu \mathrm{g}$ pGL-3 control vector with firefly luciferase reporter gene, and $100 \mathrm{nM}$ miR-302 mimics or miR-NC mixed with Lipofectamine 2000 (Invitrogen), respectively, according to the manufacturer's instructions. Luciferase activity was measured in triplicate by using the Dual Luciferase Reporter Assay System (Promega). Renilla luciferase activity was normalized to firefly luciferase activity.

Transfection. Cells were transfected by FuGENE HD (Roche) or Lipofectamine 2000 (Invitrogen) according to the manufacturer's instructions. Cells were transfected with $200 \mathrm{nM}$ of miR-302s antagomir $(50 \mathrm{nmol}$ each of miR-302a antagomir, miR-302b antagomir, miR-302c antagomir and miR-302d antagomir), $200 \mathrm{nM}$ miR-302s mimics (50 nmol each of miR-302a, miR-302b, miR-302c and miR-302d) or $200 \mathrm{nM}$ negative control NC or $200 \mathrm{nM}$ negative control antagomir as a control (Genepharma, Shanghai, China) (see Supplementary Table S5 in the Supplementary Material). Three pairs of siRNA for AKT1 and pIRES2-EGFP-AKT1 Vector (NM_005163.2) were designed and synthesized (Invitrogen). The most efficient siRNA for AKT1 were transfected into cells (see Supplementary Table S6 in the Supplementary Material) with the same procedure as miRNA transfection.

Cell cycle analysis. Cell cycle analysis was performed using the CycleTest plus DNA reagent kit (BD). Cells were collected by typsin treatment and counted with a hemocytometer. A total of 500000 cells per well were fixed, permeabilized and stained in accordance with the manufacturer's instructions, and the sample was analyzed by flow cytometry using a COULTER EPICS XL. Data were analyzed using MultiCycle software to generate the percentages of cells in G1, S and G2 to M phases of the cell cycle.

BrdU incorporation assay. Cells were incubated in a medium containing $30 \mu \mathrm{g} / \mathrm{l} \mathrm{BrdU}$ (Sigma Aldrich) for $45 \mathrm{~min}$ at $37^{\circ} \mathrm{C}$ in a humidified atmosphere $\left(5 \% \mathrm{CO}_{2}\right)$. Cells were fixed with ethanol and $50 \mathrm{mM}$ glycine, $\mathrm{pH} 2.0$, for $45 \mathrm{~min}$ at room temperature and denaturated in $4 \mathrm{M} \mathrm{HCl}$ for $10 \mathrm{~min}$. The subsequent detection of BrdU was accomplished with antibodies for BrdU (1:5000) (Sigma Aldrich). The next steps were performed according to the manufacturer's instructions using an $\mathrm{ABC}$ detection kit (Vector Laboratories, Burlingame, CA, USA).

Alkaline phosphatase staining. AP staining was performed using an Alkaline Phosphatase Detection kit (Millipore) according to the manufacturer's instructions. Cells were fixed in 90\% methanol and 10\% formamide for $2 \mathrm{~min}$ and washed once with rinse buffer (20 mM Tris- $\mathrm{HCl}, \mathrm{pH} 7.4$ and $0.05 \%$ Tween 20). Staining solution was added to the wells, and the plates were incubated in the dark for $25 \mathrm{~min}$. Bright field images were then obtained using a microscope.

Colony formation assay. hESCs were differentiated to form EBs for 15 days under non-adherent conditions. EBs were washed with PBS, trypsinized and resuspended in $\mathrm{hESC}$ media. $\mathrm{hESC}$ s were plated at 10000 cells per matrigelcoated plate. After 6 days, colonies were fixed and stained for AP. The numbers of 
a

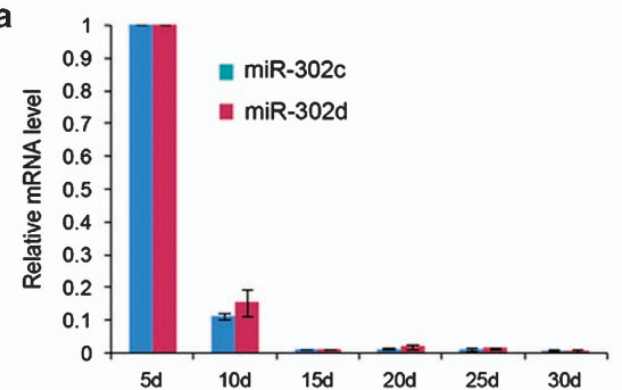

b

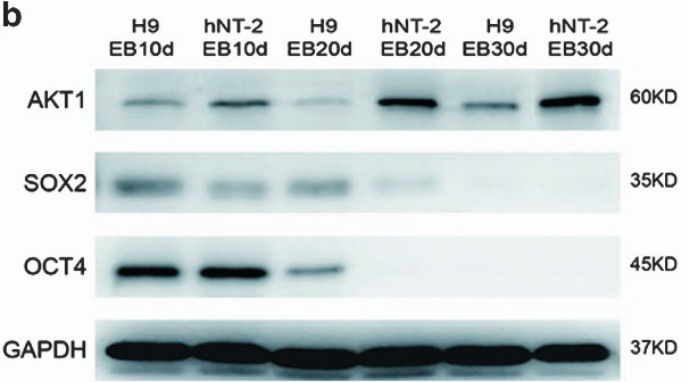

C

$\begin{array}{rrrrrr}\text { siAKT1 } & + & + & - & - & - \\ \mathrm{NCl} & - & - & + & + & + \\ \text { miR-302s } & + & - & + & - & - \\ \text { miR-NC } & - & + & - & + & + \\ \text { AKT1 } & - & - & - & - & + \\ \text { Vector } & - & - & - & - & -\end{array}$

$+\quad+$

$\begin{array}{ll}- & + \\ - & +\end{array}$

$-\quad+$

$+\quad-$

-

$+\quad+$

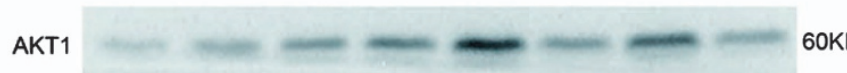

OCT4

GAPDH
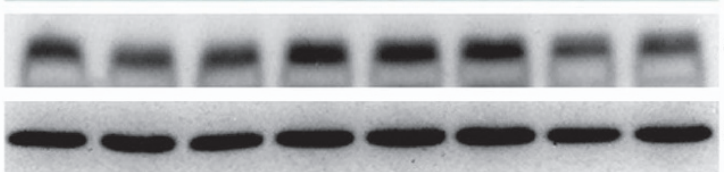

OKD

$45 \mathrm{KD}$

37KD

$\begin{array}{rllllllll}\text { siKT1 } & + & + & - & - & + & + & - & - \\ \mathrm{NCl} & - & - & + & + & - & - & + & + \\ \text { miR-302s antagomir } & + & - & + & - & + & - & + & - \\ \text { miR-NC antagomir } & - & + & - & + & - & + & - & + \\ \text { AKT1 } & - & - & - & - & - & - & + & + \\ \text { Vector } & - & - & - & - & + & + & - & -\end{array}$

AKT1

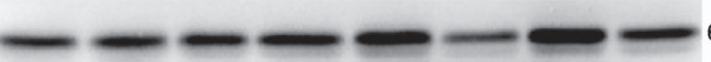

$60 K D$

OCT4

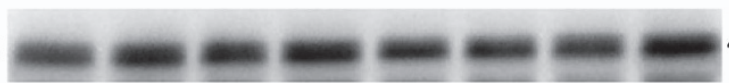

45KD

GAPDH

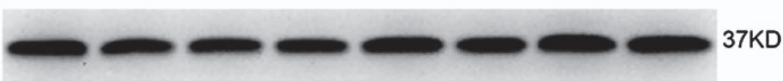

d

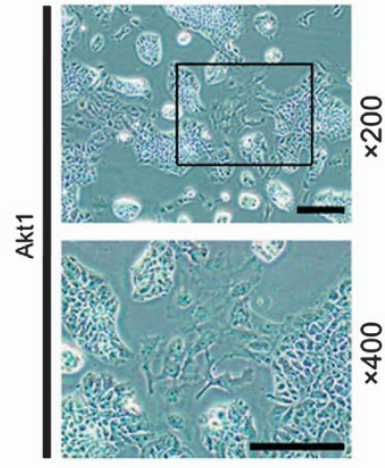

ষ্ণ

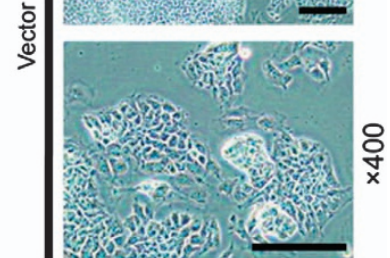

Figure 6 miR-302 contributes to the pluripotency and tumorigenicity in hPSCs by maintaining OCT4 at high expression level through suppressing of AKT1. (a) The expression of miR-302s was analyzed during the process of EB formation at days 5, 10,15,20,25 and 30 by TaqMan qRT-PCR assays. U6 was used as an internal normalization control. Data are presented as mean \pm S.D. $(n=3)$ and are representative of three independent experiments. (b) The expression of AKT1, OCT4 and SOX2 were assessed by western blot during the differentiation of hESCs and hNT-2 cells to EBs. GAPDH was used as a loading control. (c) The western blot analysis evaluated the expression of AKT1 and OCT4 when miR-302 was upregulated by transfected miR-302s mimics in AKT1 overexpressing hNT-2 cells. A negative control oligonucleotide was transfected as control, and GAPDH was used as a loading control. (d) Western blot analysis was used to evaluate the expression of AKT1 and OCT4. A negative control oligonucleotide antagomir was transfected as a control, and GAPDH was used as a loading control. (e) AKT1 overexpressing vectors were transfected into hNT-2 cells for 5 days, and the morphological changes of AKT1-overexpressed hNT-2 cells and negative control cells were observed under light microscope. Scale bars, $100 \mu \mathrm{m}$

colonies were counted and plotted as a percentage of AP-positive colonies per cell plated.

In vivo teratoma formation assay. All procedures involving mice were performed in accordance with institutional guidelines and permissions of the Ethics Committee at the Chinese Academy of Medical Sciences and Peking Union Medical College. Approximately $5 \times 10^{5} \mathrm{hNT}-2$ cells were injected subcutaneously into each 6-week-old male athymic nude (nu/nu) mouse. Xenograft sizes were measured using a vernier caliper at various time points, and the volume of the xenograft was determined using the formula $V=\left(L \times W^{2}\right) / 2$. Xenograft weights were monitored at the end point.

Immunofluorescence staining. Cells were washed twice with PBS and fixed with $4 \%$ formaldehyde (Sigma Aldrich) in PBS for 30 min at room temperature.
Then the cells were permeabilized with $0.1 \%$ TritonX-100 in PBS at room temperature for $15 \mathrm{~min}$. Next, they were blocked with 3\% BSA (Sigma Aldrich) in PBS for 30 min. Thereafter, the cells were incubated with a primary antibody against CDK2, CDK4, Cyclin D1 (1 : 50; all from Santa Cruz Biotechnology, Inc.), or SSEA4 (1:200; Millipore) at $4{ }^{\circ} \mathrm{C}$ overnight, nuclei were counterstained with Hoechst 33342 and visualized with an Olympus I 81 fluorescence microscope.

Immunohistochemical analysis. Animal or patient specimen tissues were dissected, fixed in 4\% PFA (Sigma Aldrich) in PBS overnight, processed, and sectioned into $5 \mu \mathrm{m}$ slices according to the standard procedures. Then, the sections were permeabilized with $0.1 \%$ TritonX-100 in PBS at room temperature for $15 \mathrm{~min}$, and blocked with 3\% BSA (Sigma Aldrich) in PBS for $30 \mathrm{~min}$. Then the sections were incubated with a primary antibody against OCT4 or PCNA (1:200; Cell Signaling Technology) at $4^{\circ} \mathrm{C}$ overnight. Next, the sections were treated according 
a

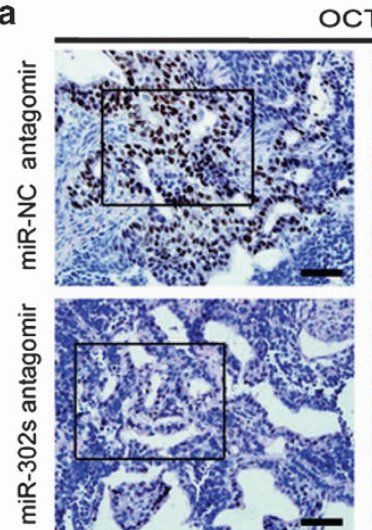

c

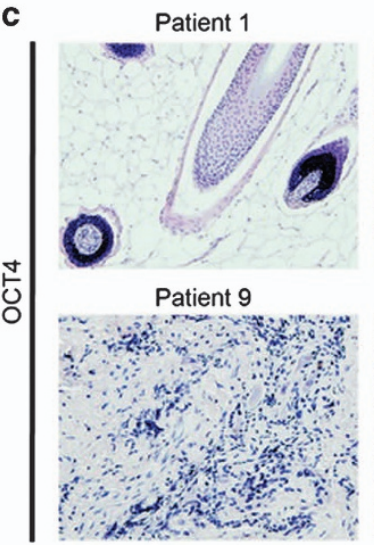

OCT4

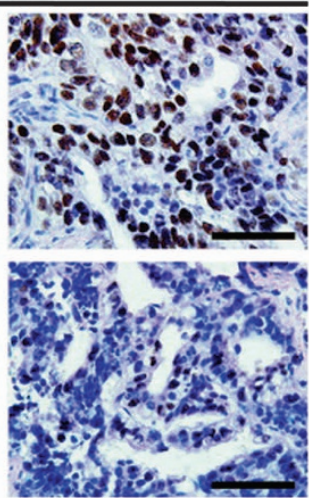

Patient 5

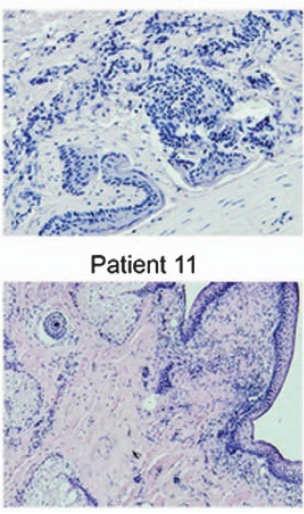

b

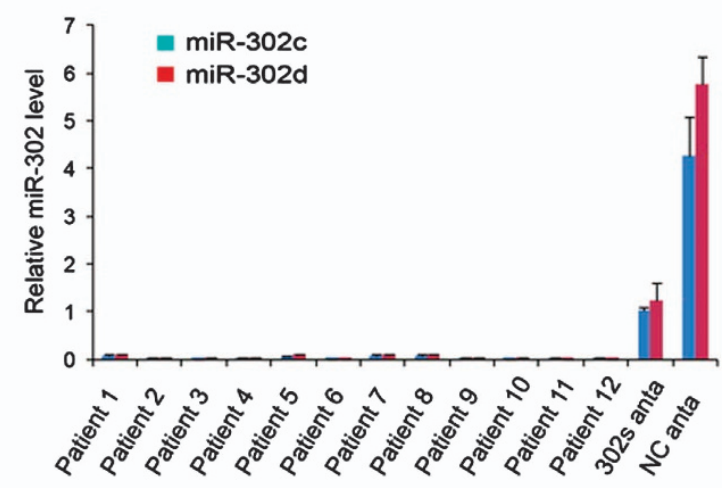

d

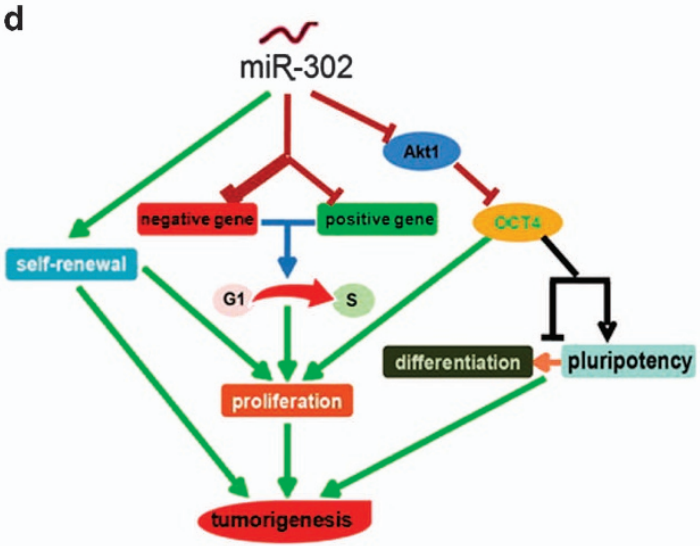

Figure 7 The expression levels of miR-302 and OCT4 in highly differentiated patient-derived teratoma and hNT-2 cells generated malignant teratoma xenografts. (a) The immunohistochemical analysis of OCT4 in miR-302s-suppressed hNT-2 cells xenografts. Negative control oligonucleotide-transfected hNT-2 cells xenografts as control. Scale bars, $100 \mu \mathrm{m}$. (b) The expression of miR-302 in 12 highly differentiated teratoma patients' specimens by TaqMan qRT-PCR analysis. RNU48 was used as an internal normalization control. The data are presented as the mean \pm S.D. $(n=3)$ and are representative of three independent experiments. miR-302s antagomir: miR-302s-suppressed hNT-2 cell xenografts, miR-NC antagomir: miR-NC-transfected hNT-2 cell xenografts. (c) The expression of OCT4 was detected in 12 highly differentiated teratoma specimens by immunohistochemistry. Scale bars, $100 \mu \mathrm{m}$. (d) The pathway by which miR-302 contributes to the tumorigenicity and the pluripotency of hPSCs through the dominant regulation of the $\mathrm{G} 1$ to $\mathrm{S}$ transition and the maintenance of the pluripotency factor OCT4 at high level via suppressing AKT1

to the manufacturer's instructions for the $\mathrm{ABC}$ detection kit (Vector Laboratories). Negative controls were performed with the omission of the primary antibody. Sections were visualized with an Olympus $B \times 51$ light microscope equipped with an Olympus DP70 camera.

Small-animal PET. 18F-FDG was synthesized automatically with a conventional module used in our clinical work in Peking Union Medical College Hospital. 18 F-FDG was pyrogen-free and qualified for clinical use with a radiochemical purity of more than $99 \%$. All mice were fasted $15 \mathrm{~h}$ before microPET scanning. The animals were intraperitoneally injected with $7.4 \mathrm{MBq}(0.2 \mathrm{mCi})$ of $18 \mathrm{~F}-\mathrm{FDG}$, allowed to regain consciousness and kept at $37^{\circ} \mathrm{C}$ until imaging. microPET scans were obtained under standardized conditions using a Siemens Inveon microPET scanner at the end study point. Tissue uptake value was expressed as radioactive count per milliliter ( $\mathrm{nCi} / \mathrm{cc}$ ). We measured interscapular BAT maximum uptake value from sagittal images and xenograft mean uptake value from coronal images. The BAT uptake ratio was then calculated for further comparison and analysis.

Statistical analysis. Two-tailed pairwise Student's $t$-tests were used to analyze the results obtained from two samples with one time point. An analysis of variance (single factor or two factors with replication) was used to compare multiple samples (at one time or several time points). Differences with $P<0.05$ were considered significant. The $P$-values were corrected for multiple testing procedures and to control for type I error rates, calculations were performed with SPSS software version 19.0 .

\section{Conflict of Interest}

The authors declare no conflict of interest.

Acknowledgements. We thank Professor Richard Boyd from Monash University for providing us $\mathrm{H} 9$ cell line. We thank Professor Hsiao Chang Chan from The Chinese University of Hong Kong for critical reading of the manuscript and giving us constructive suggestions. This work was supported by grants from the National Natural Science Foundation of China (no. 30830052), the National Key Scientific Program of China (no. 2011CB964901), the Program for Cheung Kong Scholars and Innovative Research Team in University-PCSIRT (no. IRT0909), and the National Science and Technology Major Projects for 'Drug Research and Development' (2014ZX09101042).

1. Thomson JA, Itskovitz-Eldor J, Shapiro SS, Waknitz MA, Swiergiel JJ, Marshall VS et al. Embryonic stem cell lines derived from human blastocysts. Science 1998; 282: 1145-1147.

2. Takahashi K, Tanabe K, Ohnuki M, Narita M, Ichisaka T, Tomoda K et al. Induction of pluripotent stem cells from adult human fibroblasts by defined factors. Cell 2007; 131: 861-872.

3. Przyborski SA. Differentiation of human embryonic stem cells after transplantation in immune-deficient mice. Stem Cells 2005; 23: 1242-1250.

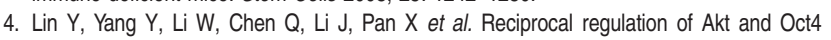
promotes the self-renewal and survival of embryonal carcinoma cells. Mol Cell 2012; 48 $627-640$. 
5. Ben-David U, Benvenisty $N$. The tumorigenicity of human embryonic and induced pluripotent stem cells. Nat Rev Cancer 2011; 11: 268-277.

6. Blum B, Benvenisty N. The tumorigenicity of human embryonic stem cells. Adv Cancer Res 2008; 100: 133-158.

7. Dreesen O, Brivanlou AH. Signaling pathways in cancer and embryonic stem cells. Stem Cell Rev 2007; 3: 7-17.

8. Burdon T, Smith A, Savatier P. Signalling, cell cycle and pluripotency in embryonic stem cells. Trends Cell Biol 2002; 12: 432-438.

9. Ghule PN, Medina R, Lengner CJ, Mandeville M, Qiao M, Dominski Z et al. Reprogramming the pluripotent cell cycle: restoration of an abbreviated $\mathrm{G} 1$ phase in human induced pluripotent stem (iPS) cells. J Cell Physiol 2011; 226: 1149-1156.

10. Becker KA, Stein JL, Lian JB, van Wijnen AJ, Stein GS. Human embryonic stem cells are pre-mitotically committed to self-renewal and acquire a lengthened G1 phase upon lineage programming. J Cell Physiol 2010; 222: 103-110.

11. Faast R, White J, Cartwright $P$, Crocker L, Sarcevic B, Dalton S. Cdk6-cyclin D3 activity in murine ES cells is resistant to inhibition by p16(INK4a). Oncogene 2004; 23 : 491-502.

12. Knoepfler PS. Deconstructing stem cell tumorigenicity: a roadmap to safe regenerative medicine. Stem Cells 2009; 27: 1050-1056.

13. Chang CC, Shieh GS, Wu P, Lin CC, Shiau AL, Wu CL. Oct-3/4 expression reflects tumor progression and regulates motility of bladder cancer cells. Cancer Res 2008; 68: 6281-6291.

14. Peng S, Maihle NJ, Huang Y. Pluripotency factors Lin28 and Oct4 identify a sub-population of stem cell-like cells in ovarian cancer. Oncogene 2010; 29: 2153-2159.

15. Hoei-Hansen CE. Application of stem cell markers in search for neoplastic germ cells in dysgenetic gonads, extragonadal tumours, and in semen of infertile men. Cancer Treat Rev 2008; 34: 348-367.

16. Zhang S, Balch C, Chan MW, Lai HC, Matei D, Schilder JM et al. Identification and characterization of ovarian cancer-initiating cells from primary human tumors. Cancer Res 2008; 68: 4311-4320.

17. Gidekel S, Pizov G, Bergman Y, Pikarsky E. Oct-3/4 is a dose-dependent oncogenic fate determinant. Cancer Cell 2003; 4: 361-370.

18. Lee J, Go Y, Kang I, Han YM, Kim J. Oct-4 controls cell-cycle progression of embryonic stem cells. Biochem J 2010; 426: 171-181.

19. Marson A, Levine SS, Cole MF, Frampton GM, Brambrink T, Johnstone S et al. Connecting microRNA genes to the core transcriptional regulatory circuitry of embryonic stem cells. Cell 2008; 134: 521-533

20. Xu N, Papagiannakopoulos T, Pan G, Thomson JA, Kosik KS. MicroRNA-145 regulates OCT4, SOX2, and KLF4 and represses pluripotency in human embryonic stem cells. Cell 2009; 137: 647-658.

21. Card DA, Hebbar PB, Li L, Trotter KW, Komatsu Y, Mishina $Y$ et al. Oct4/Sox2-regulated miR-302 targets cyclin D1 in human embryonic stem cells. Mol Cell Biol 2008; 28 : 6426-6438.

22. Wang Y, Baskerville S, Shenoy A, Babiarz JE, Baehner L, Blelloch R. Embryonic stem cellspecific microRNAs regulate the G1-S transition and promote rapid proliferation. Nat Genet 2008; 40: 1478-1483

23. Lin SL, Chang DC, Ying SY, Leu D, Wu DT. MicroRNA miR-302 inhibits the tumorigenecity of human pluripotent stem cells by coordinate suppression of the CDK2 and CDK4/6 cell cycle pathways. Cancer Res 2010; 70: 9473-9482.

24. Wilson KD, Venkatasubrahmanyam S, Jia F, Sun N, Butte AJ, Wu JC. MicroRNA profiling of human-induced pluripotent stem cells. Stem Cells Dev 2009; 18: 749-758.

25. Anokye-Danso F, Trivedi CM, Juhr D, Gupta M, Cui Z, Tian Y et al. Highly efficient miRNA mediated reprogramming of mouse and human somatic cells to pluripotency. Cell Stem Cell 2011; 8: 376-388

26. Kaid C, Silva PB, Cortez BA, Rodini CO, Semedo-Kuriki P, Okamoto OK. miR-367 promotes proliferation and stem-like traits in medulloblastoma cells. Cancer Sci 2015; 106 1188-1195.

27. Abu Dawud R, Schreiber K, Schomburg D, Adjaye J. Human embryonic stem cells and embryonal carcinoma cells have overlapping and distinct metabolic signatures. PLoS One 2012; 7: e39896.

28. Josephson R, Ording CJ, Liu Y, Shin S, Lakshmipathy U, Toumadje A et al. Qualification of embryonal carcinoma 2102Ep as a reference for human embryonic stem cell research. Stem Cells 2007; 25: 437-446.

29. Sperger JM, Chen X, Draper JS, Antosiewicz JE, Chon CH, Jones SB et al. Gene expression patterns in human embryonic stem cells and human pluripotent germ cell tumors. Proc Natl Acad Sci USA 2003; 100: 13350-13355

30. Yang $S$, Lin G, Deng L, Lu GX. Tumourigenic characteristics of embryonal carcinoma cells as a model for studying tumour progression of human embryonic stem cells. Cell Prolif 2012; 45: 299-310.

31. Andrews PW, Damjanov I, Simon D, Banting GS, Carlin C, Dracopoli NC et al. Pluripotent embryonal carcinoma clones derived from the human teratocarcinoma cell line Tera-2. Differentiation in vivo and in vitro. Lab Invest 1984; 50: 147-162.

32. Werbowetski-Ogilvie TE, Bosse M, Stewart M, Schnerch A, Ramos-Mejia V, Rouleau A et al. Characterization of human embryonic stem cells with features of neoplastic progression. Nat Biotechnol 2009; 27: 91-97.
33. Dolezalova D, Mraz M, Barta T, Plevova K, Vinarsky V, Holubcova Z et al. MicroRNAs regulate $\mathrm{p} 21$ (Waf1/Cip1) protein expression and the DNA damage response in human embryonic stem cells. Stem Cells 2012; 30: 1362-1372.

34. Liao B, Bao X, Liu L, Feng S, Zovoilis A, Liu W et al. MicroRNA cluster 302-367 enhances somatic cell reprogramming by accelerating a mesenchymal-to-epithelial transition. J Biol Chem 2011; 286: 17359-17364.

35. Borgdorff V, Lleonart ME, Bishop CL, Fessart D, Bergin AH, Overhoff MG et al. Multiple microRNAs rescue from Ras-induced senescence by inhibiting p21(Waf1/Cip1). Oncogene 2010; 29: 2262-2271.

36. Becker KA, Ghule PN, Therrien JA, Lian JB, Stein JL, van Wijnen AJ et al. Self-renewal of human embryonic stem cells is supported by a shortened G1 cell cycle phase. J Cell Physiol 2006; 209: 883-893.

37. Zhang X, Neganova I, Przyborski S, Yang C, Cooke M, Atkinson SP et al. A role for NANOG in $\mathrm{G} 1$ to $S$ transition in human embryonic stem cells through direct binding of CDK6 and CDC25A. J Cell Biol 2009; 184: 67-82.

38. Singh AM, Reynolds D, Cliff T, Ohtsuka S, Mattheyses AL, Sun $Y$ et al. Signaling network crosstalk in human pluripotent cells: a Smad2/3-regulated switch that controls the balance between self-renewal and differentiation. Cell Stem Cell 2012; 10: 312-326.

39. Watanabe S, Umehara H, Murayama K, Okabe M, Kimura T, Nakano T. Activation of Akt signaling is sufficient to maintain pluripotency in mouse and primate embryonic stem cells. Oncogene 2006; 25: 2697-2707.

40. Qi J, Yu JY, Shcherbata HR, Mathieu J, Wang AJ, Seal S et al. microRNAs regulate human embryonic stem cell division. Cell Cycle 2009; 8: 3729-3741.

41. Stadler B, Ivanovska I, Mehta K, Song S, Nelson A, Tan Y et al. Characterization of microRNAs involved in embryonic stem cell states. Stem Cells Dev 2010; 19: 935-950.

42. Bar M, Wyman SK, Fritz BR, Qi J, Garg KS, Parkin RK et al. MicroRNA discovery and profiling in human embryonic stem cells by deep sequencing of small RNA libraries. Stem Cells 2008; 26: 2496-2505.

43. Ohm JE, Mali P, Van Neste L, Berman DM, Liang L, Pandiyan $\mathrm{K}$ et al. Cancer-related epigenome changes associated with reprogramming to induced pluripotent stem cells. Cancer Res 2010; 70: 7662-7673.

44. Gutierrez-Aranda I, Ramos-Mejia V, Bueno C, Munoz-Lopez M, Real PJ, Macia A et al. Human induced pluripotent stem cells develop teratoma more efficiently and faster than human embryonic stem cells regardless the site of injection. Stem Cells 2010; 28: 1568-1570.

45. Lin SL, Chang DC, Chang-Lin S, Lin CH, Wu DT, Chen DT et al. Mir-302 reprograms human skin cancer cells into a pluripotent ES-cell-like state. RNA 2008; 14: 2115-2124.

46. Lin SL, Chang DC, Lin CH, Ying SY, Leu D, Wu DT. Regulation of somatic cell reprogramming through inducible mir-302 expression. Nucleic Acids Res 2011; 39: 1054-1065.

47. Parchem RJ, Ye J, Judson RL, LaRussa MF, Krishnakumar R, Blelloch A et al. Two miRNA clusters reveal alternative paths in late-stage reprogramming. Cell Stem Cell 2014; 14: 617-631.

48. Cai N, Wang YD, Zheng PS. The microRNA-302-367 cluster suppresses the proliferation of cervical carcinoma cells through the novel target AKT1. RNA 2013; 19: 85-95.

49. Jamshidi-Adegani F, Langroudi L, Shafiee A, Mohammadi-Sangcheshmeh A Ardeshirylajimi A, Barzegar M et al. Mir-302 cluster exhibits tumor suppressor properties on human unrestricted somatic stem cells. Tumour Biol 2014; 35: 6657-6664.

50. Fareh M, Turchi L, Virolle V, Debruyne D, Almairac F, de-la-Forest Divonne S et al. The miR 302-367 cluster drastically affects self-renewal and infiltration properties of glioma-initiating cells through CXCR4 repression and consequent disruption of the SHH-GLI-NANOG network. Cell Death Differ 2012; 19: 232-244.

51. Zhang Z, Hong Y, Xiang D, Zhu P, Wu E, Li W et al. MicroRNA-302/367 cluster governs hESC self-renewal by dually regulating cell cycle and apoptosis pathways. Stem Cell Reports 2015; 4: 645-657.

52. Kim JY, Shin KK, Lee AL, Kim YS, Park HJ, Park YK et al. MicroRNA-302 induces proliferation and inhibits oxidant-induced cell death in human adipose tissue-derived mesenchymal stem cells. Cell Death Dis 2014; 5: e1385.

53. Subramanyam D, Lamouille S, Judson RL, Liu JY, Bucay N, Derynck R et al. Multiple targets of miR-302 and miR-372 promote reprogramming of human fibroblasts to induced pluripotent stem cells. Nat Biotechnol 2011; 29: 443-448.

54. Miyoshi N, Ishii $\mathrm{H}$, Nagano $\mathrm{H}$, Haraguchi N, Dewi DL, Kano $\mathrm{Y}$ et al. Reprogramming of mouse and human cells to pluripotency using mature microRNAs. Cell Stem Cell 2011; 8 : $633-638$

55. Parchem RJ, Moore N, Fish JL, Parchem JG, Braga TT, Shenoy A et al. miR-302 is required for timing of neural differentiation, neural tube closure, and embryonic viability. Cell Rep 2015; 12: 760-773

56. Yang SL, Yang M, Herrlinger S, Liang C, Lai F, Chen JF. MiR-302/367 regulate neural progenitor proliferation, differentiation timing, and survival in neurulation. Dev Biol 2015; 408: 140-150.

57. Wade SL, Langer LF, Ward JM, Archer TK. MiRNA-mediated regulation of the SWI/SNF chromatin remodeling complex controls pluripotency and endodermal differentiation in human ESCs. Stem Cells 2015; 33: 2925-2935. 
58. Yilmaz $\mathrm{OH}$, Valdez R, Theisen BK, Guo W, Ferguson DO, Wu H et al. Pten dependence distinguishes haematopoietic stem cells from leukaemia-initiating cells. Nature 2006; 441: 475-482.

59. Sykes SM, Lane SW, Bullinger L, Kalaitzidis D, Yusuf R, Saez B et al. AKT/FOXO signaling enforces reversible differentiation blockade in myeloid leukemias. Cell 2011; 146: 697-708.

60. Tothova Z, Kollipara R, Huntly BJ, Lee BH, Castrillon DH, Cullen DE et al. FoxOs are critical mediators of hematopoietic stem cell resistance to physiologic oxidative stress. Cell 2007; 128: 325-339.

61. Niwa $H$, Ogawa $K$, Shimosato $D$, Adachi K. A parallel circuit of LIF signalling pathways maintains pluripotency of mouse ES cells. Nature 2009; 460: 118-122.

62. Kharas MG, Okabe R, Ganis JJ, Gozo M, Khandan T, Paktinat M et al. Constitutively active AKT depletes hematopoietic stem cells and induces leukemia in mice. Blood 2010; 115: $1406-1415$.

63. Chen B, Xue Z, Yang G, Shi B, Yang B, Yan Y et al. Akt-signal integration is involved in the differentiation of embryonal carcinoma cells. PLoS One 2013; 8: e64877.

64. Bussolati B, Bruno S, Grange C, Ferrando U, Camussi G. Identification of a tumor-initiating stem cell population in human renal carcinomas. FASEB $J$ 2008; 22: 3696-3705.
65. Chiou SH, Wang ML, Chou YT, Chen CJ, Hong CF, Hsieh WJ et al. Coexpression of Oct4 and Nanog enhances malignancy in lung adenocarcinoma by inducing cancer stem cell-like properties and epithelial-mesenchymal transdifferentiation. Cancer Res 2010; 70: 10433-10444.

66. Le Blanc K, Mougiakakos D. Multipotent mesenchymal stromal cells and the innate immune system. Nat Rev Immunol 2012; 12: 383-396.

(c) (i) Cell Death and Disease is an open-access journal published by Nature Publishing Group. This work is licensed under a Creative Commons Attribution 4.0 International License. The images or other third party material in this article are included in the article's Creative Commons license, unless indicated otherwise in the credit line; if the material is not included under the Creative Commons license, users will need to obtain permission from the license holder to reproduce the material. To view a copy of this license, visit http://creativecommons.org/licenses/by/4.0/

Supplementary Information accompanies this paper on Cell Death and Disease website (http://www.nature.com/cddis) 